\title{
Spectra, spectra, spectra - Tensor triangular spectra versus Zariski spectra of endomorphism rings
}

\author{
PAUL BALMER
}

\begin{abstract}
We construct a natural continuous map from the triangular spectrum of a tensor triangulated category to the algebraic Zariski spectrum of the endomorphism ring of its unit object. We also consider graded and twisted versions of this construction. We prove that these maps are quite often surjective but far from injective in general. For instance, the stable homotopy category of finite spectra has a triangular spectrum much bigger than the Zariski spectrum of $\mathbb{Z}$. We also give a first discussion of the spectrum in two new examples, namely equivariant $K K$-theory and stable $\mathbb{A}^{1}-$ homotopy theory.
\end{abstract}

18E30; 14F05, 19K35, 20C20, 55P42, 55U35

\section{Introduction}

Algebraic geometers, stable topologists, finite group representation theorists, motivic theorists, noncommutative geometers and many other mathematicians have triangulated categories in common: The derived category of sheaves of modules over a scheme, the stable homotopy category of topological spectra, the derived category or the stable category of representations of a finite group or finite group scheme, the various motivic derived categories, Morel and Voevodsky's stable $\mathbb{A}^{1}$-homotopy category and equivariant $K K$-theory or $E$-theory of $C^{*}$-algebras are famous examples. In several cases, a tensor structure is also available and is especially well-behaved on the triangulated subcategory of compact objects. In the above examples, this leads us to focus on perfect complexes, finite topological spectra, finite dimensional representations, geometric motives, etc.

This profusion of examples motivates the study of tensor triangulated categories per se. Emphasizing the geometric aspects of this unified theory leads us to a subject called tensor triangular geometry, to which the present paper belongs.

To explain these ideas, let us denote by $\mathscr{K}$ one of our tensor triangulated categories (say, of compact objects), by $\otimes: \mathscr{K} \times \mathscr{K} \longrightarrow \mathscr{K}$ the symmetric tensor $x \otimes y=y \otimes x$ and by $\mathbb{1} \in \mathscr{K}$ the $\otimes$-unit: $\mathbb{1} \otimes x=x$. 
We began our geometric study in [1] with the definition of a topological space

$$
\operatorname{Spc}(\mathscr{K})
$$

called the spectrum of $\mathscr{Y}$ (see also Definition 1.3 below). We call it the triangular spectrum here, to avoid confusion with other meanings of the word "spectrum". This fundamental space $\operatorname{Spc}(\mathscr{K})$ is the canvas on which to draw tensor triangular geometry. For instance, every object $x \in \mathscr{K}$ has a support, $\operatorname{supp}(x) \subset \operatorname{Spc}(\mathscr{K})$, which is a closed subset behaving nicely with respect to exact triangles and tensor product. The pair $(\operatorname{Spc}(\mathscr{K})$, supp) is indeed the best such pair, for it has a universal property: In the language of [1, Theorem 3.2], ( $\operatorname{Spc}(\mathscr{K}), \operatorname{supp})$ is "the final support datum".

Although a young subject, tensor triangular geometry has already produced some interesting results. For instance, in [2, Theorem 2.11], we proved that if an object $x \in \mathscr{K}$ has a disconnected support, ie $\operatorname{supp}(x)=Y_{1} \cup Y_{2}$ with $Y_{1}$ and $Y_{2}$ closed and disjoint, then the object is decomposable accordingly: $x \simeq x_{1} \oplus x_{2}$ with $\operatorname{supp}\left(x_{i}\right)=Y_{i}$. This neat result has applications, like the gluing technique of Balmer-Favi [6] and its representation theoretic incarnations in Balmer-Benson-Carlson [5] and in our paper [4]. Another application, this time to algebraic $K$-theory of schemes, can be found in our paper [3].

In all applications though, the crucial anchor point is the computation of the triangular spectrum $\operatorname{Spc}(\mathscr{K})$ in the first place. Without this knowledge, abstract results of tensor triangular geometry are difficult to translate into concrete terms. It is therefore a major challenge to compute the spectrum $\operatorname{Spc}(\mathscr{K})$ in as many examples as possible, or at least to provide some information about that space when the full determination of $\operatorname{Spc}(\mathscr{K})$ lies beyond reach of the community's current forces.

Actually, by our paper [1, Theorem 4.10, Theorem 5.2] and Buan, Krause and Solberg [9, Corollary 5.2], we know that the information about $\mathscr{K}$ contained in the space $\operatorname{Spc}(\mathscr{K})$ is exactly equivalent to the so-called classification of thick triangulated $\otimes$-ideals of $\mathscr{K}$. Existing such classifications allow us to compute $\operatorname{Spc}(\mathscr{K})$ and, conversely, given the space $\operatorname{Spc}(\mathscr{K})$, we obtain a classification of thick triangulated $\otimes$-ideals by assigning to suitable subsets $Y \subset \operatorname{Spc}(\mathscr{K})$ the thick triangulated $\otimes$-ideal $\mathscr{K}_{Y}:=\{a \in \mathscr{K} \mid \operatorname{supp}(a) \subset$ $Y\}$ of $\mathscr{Y}$.

Using preexisting classifications of thick $\otimes$-ideals, some triangular spectra have been computed in [1;9]: In algebraic geometry, the spectrum of the derived category of perfect complexes $\mathscr{K}=\mathrm{D}^{\text {perf }}(X)$ over a quasicompact and quasiseparated scheme $X$ reconstructs the scheme $X$ itself; this uses Thomason's classification, initiated by Hopkins and Neeman in the affine case. In modular representation theory, the spectrum of the stable category $\mathscr{K}=k G$-stab is the projective support variety $\mathcal{V}_{G}(k)=\operatorname{Proj}\left(\mathrm{H}^{\bullet}(G, k)\right)$, 
by Benson, Carlson and Rickard's classification, generalized to finite group schemes by Friedlander and Pevtsova. See our paper [1] for details and references about these statements. Recently, Krishna [17] also identified spectra of perfect complexes over Deligne-Mumford stacks as coarse moduli spaces.

Yet, the precursor of all these classifications is to be found in topology, in the celebrated work of Hopkins and Smith [15]. Since the reformulation of this original result in terms of a triangular spectrum has not been written down so far, we provide it in Section 9, en passant; see also (0-1) further down in this introduction.

Such classifications are usually rather nontrivial. In noncommutative topology little is known beyond the very first cases, recently treated by Dell' Ambrogio in [11] (see Example 8.7 below). In $\mathbb{A}^{1}$-homotopy theory, the classification is not known and the question is essentially wide open. It would be very interesting to use the theory of triangular spectra in order to compute the spectrum directly, by independent techniques and then deduce the classification by [1, Theorem 4.10]. This is actually sometimes possible. It works for instance in commutative algebra, as we shall see in Remark 8.4. But more generally, in the new areas where the classification might be too hard for now, any information about the relevant triangular spectra provides an indication of the complexity of the triangulated categories involved. Such results are among the objectives of the present paper, as we shall see below.

Let us stress the point that, to this day, all known homeomorphisms between $\operatorname{Spc}(\mathscr{K})$ and a more explicit topological space $X$ come from the universal property of the pair $(\operatorname{Spc}(\mathscr{K})$, supp) as a final support datum. Such maps $X \rightarrow \operatorname{Spc}(\mathscr{K})$ are always going from a candidate-spectrum $X$ towards $\operatorname{Spc}(\mathscr{K})$. The main purpose of the present article is to construct continuous maps out of $\operatorname{Spc}(\mathscr{K})$, into topological spaces $X$ of "spectral" nature: $\operatorname{Spc}(\mathscr{K}) \rightarrow X$.

Indeed, by many aspects, the triangular spectrum $\operatorname{Spc}(\mathscr{K})$ resembles the Zariski spectrum of a commutative ring; first naively, since points of $\operatorname{Spc}(\mathscr{K})$ are sophisticated prime ideals; see Definition 1.3. But more rigourously, the space $\operatorname{Spc}(\mathscr{K})$ is always spectral in the sense of Hochster [14], ie it has a basis of quasicompact opens and any irreducible closed has a unique generic point. Hochster proved that this characterizes spectra of commutative rings among topological spaces. So, we might hope for a nice little commutative ring $R$ such that $\operatorname{Spc}(\mathscr{K})=\operatorname{Spec}(R)$. Since the endomorphism ring of the unit, $\operatorname{End}_{\mathscr{K}}(\mathbb{1})$, provides a natural commutative ring for each tensor triangulated category $\mathscr{K}$, it is legitimate to ask whether the triangular spectrum $\operatorname{Spc}(\mathscr{K})$ can be related to the Zariski spectrum $\operatorname{Spec}\left(\operatorname{End}_{\mathscr{K}}(\mathbb{1})\right)$. Alternatively, one can consider the homogeneous spectrum of the graded endomorphism $\operatorname{ring} \operatorname{End}_{\mathscr{K}}^{\bullet}(\mathbb{1})=\operatorname{Hom}_{\mathcal{K}}\left(\mathbb{1}, \Sigma^{\bullet}(\mathbb{1})\right)$. Our main construction establishes such links: 
Theorem (Theorem 5.3 and Corollary 5.6) There exist two continuous maps

$$
\begin{aligned}
& \rho_{\mathscr{K}}: \operatorname{Spc}(\mathscr{K}) \longrightarrow \operatorname{Spec}\left(\operatorname{End}_{\mathscr{K}}(\mathbb{1})\right), \\
& \rho_{\mathscr{K}}^{\bullet}: \operatorname{Spc}(\mathscr{K}) \longrightarrow \operatorname{Spec}^{\mathrm{h}}\left(\operatorname{End}_{\mathscr{K}}^{\bullet}(\mathbb{1})\right)
\end{aligned}
$$

which are natural in the tensor triangulated category $\mathscr{K}$.

A direct consequence is that one can produce open covers of the triangular spectrum $\operatorname{Spc}(\mathscr{K})$ by taking preimages of open covers of the above Zariski spectra, even before knowing $\operatorname{Spc}(\mathscr{K})$. This will be investigated in subsequent work.

It is interesting to inspect the various incarnations of the maps $\rho_{\mathscr{K}}$ and $\rho_{\mathscr{K}}^{\bullet}$ in the areas of application of tensor triangular geometry mentioned above. These maps are always nontrivial but seem especially informative in algebraic examples. In fact, the maps $\rho_{\mathscr{r}}$ and $\rho_{\mathscr{K}}^{\bullet}$ are often surjective (yet not always; see Example 8.3):

Theorem (Theorem 7.13) Assume that $\mathscr{K}$ is connective, ie $\operatorname{Hom}_{\mathscr{K}}\left(\Sigma^{i}(\mathbb{1}), \mathbb{1}\right)=0$ for all $i<0$. Then the map $\rho_{\mathscr{K}}: \operatorname{Spc}(\mathscr{K}) \longrightarrow \operatorname{Spec}\left(\operatorname{End}_{\mathscr{K}}(\mathbb{1})\right)$ is surjective.

There is also a rather mild sufficient condition for surjectivity in the graded case:

Theorem (Theorem 7.3 and Corollary 7.4) If the graded ring $\operatorname{End}_{\mathscr{K}}^{\bullet}(\mathbb{1})$ is coherent, for instance if it is noetherian, then both maps $\rho_{\mathscr{K}}^{\bullet}: \operatorname{Spc}(\mathscr{K}) \longrightarrow \operatorname{Spec}^{\mathrm{h}}\left(\operatorname{End}_{\mathscr{K}}^{\bullet}(\mathbb{1})\right)$ and $\rho_{\mathscr{K}}: \operatorname{Spc}(\mathscr{K}) \longrightarrow \operatorname{Spec}\left(\operatorname{End}_{\mathscr{K}}(\mathbb{1})\right)$ are surjective.

On the other hand, these maps $\rho_{\mathscr{K}}$ and $\rho_{\mathscr{K}}^{\bullet}$ are not injective in general, already in algebraic geometry, that is, for $\mathscr{K}=\mathrm{D}^{\text {perf }}(X)$ over a scheme $X$. For instance, in the case of projective $n$-space $X=\mathbb{P}_{k}^{n}$, we have $\operatorname{Spc}(\mathscr{K}) \cong \mathbb{P}_{k}^{n}$ but $\operatorname{End}_{\mathscr{K}}(\mathbb{1})=\operatorname{End}_{\mathscr{K}}^{\bullet}(\mathbb{1})=k$. However, such counterexamples are rather weak for the following two reasons. First, when $X=\operatorname{Spec}(A)$ is affine, the map $\rho_{\mathscr{K}}^{\bullet}=\rho_{\mathscr{K}}$ is actually a homeomorphism (Proposition 8.1), inverse to the one $\operatorname{Spec}(A) \stackrel{\sim}{\rightarrow} \operatorname{Spc}(\mathscr{K})$ given in [1, Corollary 5.6]. So, in algebraic geometry, $\rho_{\mathscr{K}}^{\bullet}$ is at least "locally injective". Secondly, in examples like the above $X=\mathbb{P}_{k}^{n}$, one can in fact obtain injectivity of $\rho_{\mathscr{K}}^{\bullet}$ if we twist the graded endomorphism ring by a line bundle; see Remark 8.2.

In topology, for $\mathscr{K}=\mathrm{SH}^{\text {fin }}$ the (Spanier-Whitehead) stable homotopy category of finite spectra, the triangular spectrum $\mathrm{Spc}\left(\mathrm{SH}^{\mathrm{fin}}\right)$ and the surjective continuous map 
$\rho_{\mathrm{SH}} \mathrm{fin}^{\mathrm{in}} \mathrm{Spc}\left(\mathrm{SH}^{\mathrm{fin}}\right) \longrightarrow \operatorname{Spec}(\mathbb{Z})$ look as follows (see Corollary 9.5):

$(0-1)$

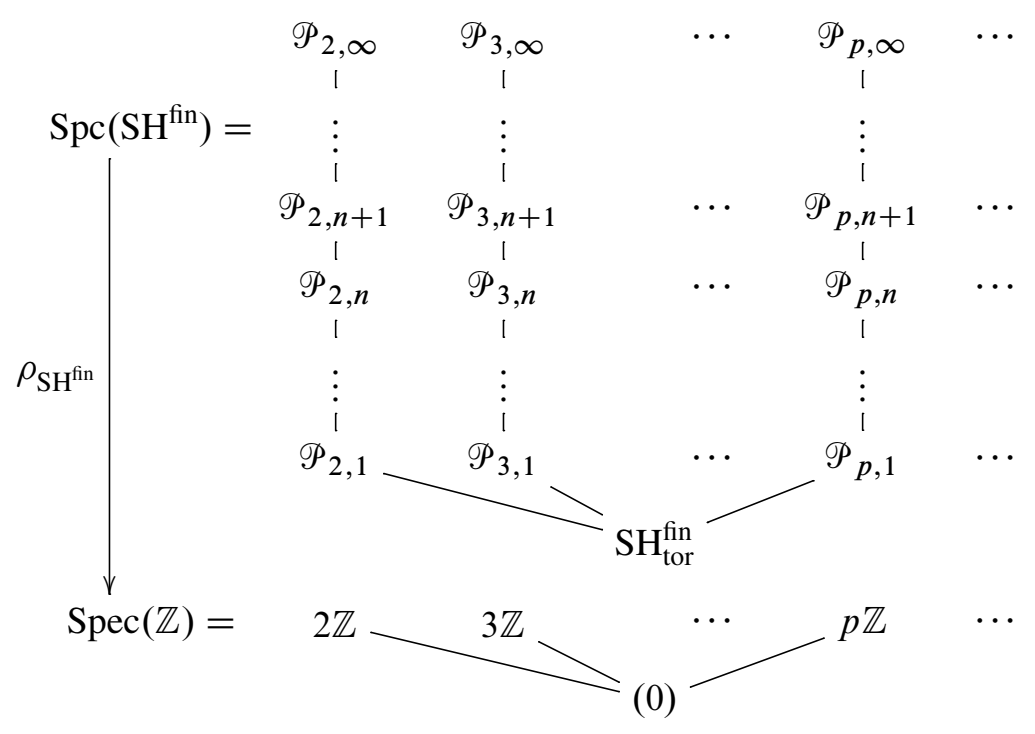

Here, $p$ runs through prime numbers and $n$ through positive integers. The notation in this result (the announced reformulation of Hopkins and Smith's classification) is explained in Section 9. The map $\rho_{\mathrm{SH}}{ }^{\text {fin }}$ is the obvious vertical projection. Note that its noninjectivity is more serious than before. Indeed, here, injectivity even fails locally, that is, for $\mathrm{SH}_{(p)}^{\mathrm{fin}}$ the localization of $\mathrm{SH}^{\mathrm{fin}}$ at a prime $p$, despite $\mathrm{SH}_{(p)}^{\mathrm{fin}}$ being a local tensor triangulated category (in the sense of Section 4).

This simply indicates that endomorphism rings of the unit cannot control the whole triangulated category $\mathscr{K}$ and that $\operatorname{Spc}(\mathscr{K})$ is a richer invariant.

At this stage, I do not know a good general criterion which could guarantee the (local) injectivity of the map $\rho_{\mathscr{K}}: \operatorname{Spc}(\mathscr{K}) \rightarrow \operatorname{Spec}\left(\operatorname{End}_{\mathscr{K}}(\mathbb{1})\right)$ or its twisted graded version $\rho_{\mathscr{K}}^{\bullet}$. Such a criterion should probably work only for "algebraic enough" triangulated categories $\mathscr{K}$ and seems to be an interesting research challenge.

Finally, here are the announced applications to $\mathbb{A}^{1}$-homotopy theory and noncommutative topology, where the triangular spectrum is not known yet but some partial information can be obtained by the techniques of this paper:

Theorem (Corollary 10.1) Let $\mathscr{K}^{\mathbb{A}^{1}}:=\left(\mathrm{SH}_{F}^{\mathbb{A}^{1}}\right)^{\mathrm{c}}$ be the subcategory of compact objects in the stable $\mathbb{A}^{1}$-homotopy category $\mathrm{SH}_{F}^{\mathrm{A}^{F}}$ over a perfect field $F$ of characteristic different from 2. Let $\mathrm{GW}(F)$ be the Grothendieck-Witt ring of quadratic forms over $F$. Then there is a surjective continuous map $\rho_{\mathscr{K}^{\mathbb{A}}}: \operatorname{Spc}\left(\mathscr{K}^{\mathbb{A}^{1}}\right) \longrightarrow \operatorname{Spec}(\operatorname{GW}(F))$. 
This uses among other things our first criterion for surjectivity (connectivity). The Zariski spectrum $\operatorname{Spec}(\mathrm{GW}(F))$ is classical and can be found in Remark 10.2.

Theorem (Corollary 8.8) Let $G$ be a compact Lie group and $K K^{G}$ be the category of separable $G-C^{*}$-algebras with morphisms given by Kasparov's equivariant $K K-$ theory (see Meyer [23]). Let $R(G):=K_{0}^{G}(\mathbb{C})$ be the complex representation ring of $G$. Then there is a continuous surjection $\rho_{K K^{G}}: \operatorname{Spc}\left(K K^{G}\right) \longrightarrow \operatorname{Spec}(R(G))$.

This uses among other things our second criterion for surjectivity (noetherianity). The Zariski spectrum $\operatorname{Spec}(R(G))$ is classical and can be found in Segal [31, Section 3].

The structure of the paper is the following. After quickly recalling from [1] a few facts about tensor triangulated categories and their spectrum in the one-page Section 1, we consider the central ring $\mathrm{R}_{\mathscr{K}}=\operatorname{End}_{\mathscr{K}}(\mathbb{1})$ in Section 2 . As in any symmetric monoidal category, the ring $\mathrm{R}_{\mathscr{K}}$ acts on any object of $\mathscr{K}$. We show in Theorem 2.15 that the smallest thick triangulated $\otimes$-ideal $\langle\operatorname{cone}(f)\rangle$ containing the cone of an endomorphism $f: \mathbb{1} \rightarrow \mathbb{1}$ coincides with the subcategory of objects $x \in \mathscr{K}$ on which $f$ is nilpotent. In Section 3, we use these techniques to construct localizations $S^{-1} \mathscr{K}$ of any tensor triangulated category $\mathscr{K}$, with respect to any multiplicative subset $S \subset \mathrm{R}_{\mathscr{K}}$. We also provide graded versions of these constructions.

Section 4 has a broader importance for tensor triangular geometry, for we introduce the notion of local tensor triangulated category. For instance, a rigid (Definition 1.5) tensor triangulated category $\mathscr{K}$ is local if and only if

$$
x \otimes y=0 \text { implies } \quad x=0 \text { or } y=0 .
$$

Following commutative algebra, one might naively call tensor triangulated categories satisfying (0-2) "integral" or "domains". The correct terminology, namely "local", is justified by the fact that $\operatorname{Spc}(\mathscr{K})$ is a local topological space (Proposition 4.2) and comforted by the fact that a commutative ring $A$ is local if and only if $\mathrm{D}^{\text {perf }}(A)$ is local in the above sense. A key result says:

Theorem (Theorem 4.5) If $\mathscr{K}$ is a local tensor triangulated category, then $\mathrm{R}_{\mathscr{K}}$ is a local ring and $\mathrm{R}_{\mathscr{K}}^{\bullet}=$ End $_{\mathscr{K}}^{\bullet}(\mathbb{1})$ is a local graded ring.

It is then easy to construct the maps $\rho_{\mathscr{K}}$ and $\rho_{\mathscr{K}}^{\bullet}$ explicitly in Section 5. Somewhat independent of the rest, Section 6 recalls from [1] the sheaf of rings $\mathcal{O}_{\mathscr{K}}$ on the spectrum $\operatorname{Spc}(\mathscr{K})$. This yields a locally ringed space $\operatorname{Spec}(\mathscr{K}):=\left(\operatorname{Spc}(\mathscr{K}), \mathcal{O}_{\mathscr{K}}\right)$ and our maps $\rho_{\mathscr{K}}$ and $\rho_{\mathscr{K}}^{\bullet}$ become morphisms of locally ringed spaces. Incidentally, the example of $\mathscr{K}=\mathrm{SH}^{\mathrm{fin}}$ also shows that the locally ringed space $\operatorname{Spec}(\mathscr{K})$ is not a scheme in 
general. We prove our surjectivity results in Section 7. The last three sections are dedicated to examples, from algebraic geometry, modular representation theory and $K K$-theory in Section 8, from topological stable homotopy theory in Section 9 and finally from stable $\mathbb{A}^{1}$-homotopy theory in Section 10.

Acknowledgments I warmly thank Aravind Asok, Mark Blunk, Theo Bühler, Ivo Dell'Ambrogio, Fabien Morel, Amnon Neeman, Manuel Ojanguren, Tobias Peter, Greg Stevenson and Charles Walter for precious discussions and comments. I also thank the referee for suggesting some improvements.

This research was supported by NSF grant 0654397.

\section{Basics}

Convention 1.1 Unless stated otherwise, all categories are assumed essentially small and all subcategories are assumed full and closed under isomorphism.

Definition 1.2 A tensor triangulated category $(\mathscr{K}, \otimes, \mathbb{1})$ is the data of a triangulated category $\mathscr{K}$ and of a "compatible" symmetric monoidal structure

$$
\otimes: \mathscr{K} \times \mathscr{K} \longrightarrow \mathscr{K}
$$

with unit $\mathbb{1} \in \mathscr{K}$ (for symmetric monoidal; see Mac Lane [21, Section XI.1]). We denote by $\sigma_{a, b}: a \otimes b \stackrel{\sim}{\rightarrow} b \otimes a$ the symmetry isomorphism (switch). The compatibility means that $\otimes$ should be exact in each variable. See further references in [1, Definition 1.1].

Definition 1.3 In [1], we define the spectrum, $\operatorname{Spc}(\mathscr{K})$, of a tensor triangulated category $\mathscr{K}$ as the set of proper thick triangulated $\otimes$-ideals $\mathscr{P} \subsetneq \mathscr{K}$ which are prime, ie such that $a \otimes b \in \mathscr{P}$ implies $a$ or $b \in \mathscr{P}$. We define for all $a \in \mathscr{K}$ the open subsets $U(a):=\{\mathscr{P} \in \operatorname{Spc}(\mathscr{K}) \mid a \in \mathscr{P}\}$, which form a basis of the topology on $\operatorname{Spc}(\mathscr{K})$. The support of an object $a \in \mathscr{K}$ is the closed complement of $U(a)$ :

$$
\operatorname{supp}(a)=\{\mathscr{P} \in \operatorname{Spc}(\mathscr{K}) \mid a \notin \mathscr{P}\} \subset \operatorname{Spc}(\mathscr{K})
$$

Remark 1.4 Let $\mathscr{F} \subset \mathscr{K}$ be a thick triangulated $\otimes$-ideal (that is, $\mathscr{F} \subset \mathscr{K}$ is a nonempty full subcategory, stable in $\mathscr{K}$ under taking cones, direct summands and tensor product with any object of $\mathscr{K})$. The Verdier quotient $\mathscr{K} / \mathscr{\mathscr { S }}$ [32, Section II.2] becomes a $\otimes-$ triangulated category, with $q: \mathscr{K} \rightarrow \mathscr{K} / \mathscr{\mathscr { F }}$ being $\otimes$-triangulated. The spectrum of the quotient $\operatorname{Spc}(\mathscr{K} / \mathscr{F})$ is naturally homeomorphic to the subspace $\{\mathscr{P} \in \operatorname{Spc}(\mathscr{K}) \mid \mathscr{P} \supset \mathscr{F}\}$ of $\operatorname{Spc}(\mathscr{K})$, via $2 \mapsto q^{-1}(2)$; see [1, Proposition 3.11]. 
Definition 1.5 We say that the tensor triangulated category is rigid if there exists an exact functor $D: \mathscr{K}^{\mathrm{op}} \rightarrow \mathscr{K}$ and natural isomorphisms

$$
\operatorname{Hom}_{\mathscr{K}}(a \otimes b, c) \simeq \operatorname{Hom}_{\mathscr{K}}(a, D(b) \otimes c) .
$$

This says that $\underline{\operatorname{hom}}(b, c):=D(b) \otimes c$ is an internal hom functor. In [2; 6] we wrote "strongly closed" instead of "rigid" but the latter terminology seems more common.

Notation 1.6 For a collection $\mathcal{E} \subset \mathscr{K}$ of objects, we denote by $\langle\mathcal{E}\rangle$ the smallest thick triangulated $\otimes$-ideal containing $\mathcal{E}$.

Proposition 1.7 Let $\mathcal{E} \subset \mathscr{K}$ be a class of objects in our $\otimes$-triangulated category $\mathscr{K}$.

(a) For $a \in\langle\mathcal{E}\rangle$ and for $b \in \mathscr{K}$, we have $a \otimes b \in\langle\mathcal{E} \otimes b\rangle$.

(b) If $\langle\mathcal{E}\rangle=\mathscr{K}$, then for every $n \geq 1$ we have $\left\langle\left\{x^{\otimes n} \mid x \in \mathcal{E}\right\}\right\rangle=\mathscr{K}$ as well.

Proof (a) The subcategory $\{x \in \mathscr{K} \mid x \otimes b \in\langle\mathcal{E} \otimes b\rangle\}$ is a thick triangulated $\otimes$-ideal containing $\mathcal{E}$, hence containing $\langle\mathcal{E}\rangle$, hence containing $a$.

(b) There exists $x_{1}, \ldots, x_{m} \in \mathcal{E}$ such that $\mathbb{1} \in\left\langle x_{1}, \ldots, x_{m}\right\rangle$. So, we can assume $\mathcal{E}=\left\{x_{1}, \ldots, x_{m}\right\}$ finite. From (a), we get $x_{i} \in\left\langle\mathcal{E} \otimes x_{i}\right\rangle \subset\langle\mathcal{E}(2)\rangle$, where $\mathcal{E}(r):=$ $\left\{y_{1} \otimes \cdots \otimes y_{r} \mid y_{1}, \ldots, y_{r} \in \mathcal{E}\right\}$ for any $r \geq 2$. Hence $\mathbb{1} \in\left\langle x_{1}, \ldots, x_{m}\right\rangle \subset\langle\mathcal{E}(2)\rangle$. Repeating this, we get $\mathbb{1} \in\langle\mathcal{E}(r)\rangle$ for all $r \geq 2$. Now since $\mathcal{E}$ is finite, for any given $n \geq 1$, there exists $r \geq 1$ big enough such that $\mathcal{E}(r) \subset\left\langle\left\{x^{\otimes n} \mid x \in \mathcal{E}\right\}\right\rangle$. Hence $\mathbb{1} \in\left\langle\left\{x^{\otimes n} \mid x \in \mathcal{E}\right\}\right\rangle$ and therefore $\mathscr{K}=\left\langle\left\{x^{\otimes n} \mid x \in \mathcal{E}\right\}\right\rangle$.

\section{Morphisms and actions}

Definition 2.1 For a tensor triangulated category $(\mathscr{K}, \otimes, \mathbb{1})$, we define its central ring $\mathrm{R}_{\mathscr{K}}$ to be the endomorphism ring of its unit

$$
\mathrm{R}_{\mathscr{K}}:=\operatorname{End}_{\mathscr{K}}(\mathbb{1}) .
$$

It is commutative since composition coincides with tensor product:

Proposition 2.2 For all $a, b \in \mathscr{K}$, the group $\operatorname{Hom}_{\mathscr{K}}(a, b)$ is a left $\mathrm{R}_{\mathscr{K}}$-module via $(f, g) \mapsto f \otimes g$, for $f \in \mathrm{R}_{\mathscr{K}}$ and $g \in \operatorname{Hom}_{\mathscr{K}}(a, b)$, using the identifications $\mathbb{1} \otimes a \cong a$ and $\mathbb{1} \otimes b \cong b$. This left action coincides with the right action $(g, f) \mapsto g \otimes f$ defined analogously. We denote this action by $f \cdot g$. With this structure, composition $\operatorname{Hom}_{\mathscr{K}}(b, c) \times \operatorname{Hom}_{\mathscr{K}}(a, b) \rightarrow \operatorname{Hom}_{\mathscr{K}}(a, c),(g, h) \mapsto g \circ h$, becomes $\mathrm{R}_{\mathscr{K}}$-bilinear. 
Proof Let us check that $f \otimes g=g \otimes f$. Consider the commutative diagram:

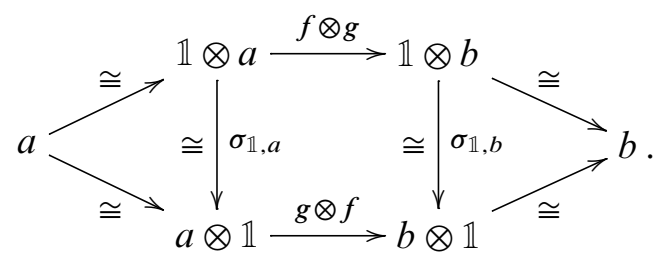

The left and right triangles commute by axiom of symmetric monoidal categories [21, XI.1.(4), page 252]. The upper composition from $a$ to $b$ is what we called $f \otimes g$ and the lower one is $g \otimes f$. The rest of the proof is left to the reader (or see [11, 2.1.1]).

Remark 2.3 Proposition 2.2 justifies the term "central ring" for the ring R F $_{\mathscr{C}}$.

Definition 2.4 An object $u \in \mathscr{K}$ is invertible if $u \otimes v \simeq \mathbb{1}$ for some $v \in \mathscr{K}$. When $\mathscr{K}$ is rigid (Definition 1.5), $v$ is necessarily isomorphic to $D(u)$.

Example 2.5 When $u$ is invertible, so is $\Sigma^{i}(u)$ for every $i \in \mathbb{Z}$. In particular, $\Sigma^{i}(\mathbb{1})$ is invertible for every $i \in \mathbb{Z}$, with inverse $\Sigma^{-i}(\mathbb{1})$.

Notation 2.6 For a morphism $f: \mathbb{1} \rightarrow u$ and for an object $a \in \mathscr{K}$, we denote by

$$
f \mathrm{~h}_{a}: a \longrightarrow u \otimes a
$$

the morphism $f \otimes \operatorname{id}_{a}$ (composed with $a \cong \mathbb{1} \otimes a$ ). This applies in particular to $u=\mathbb{1}$, in which case, $f \vdash_{a}$ is nothing but $f \cdot \mathrm{id}_{a}$ in the notation of Proposition 2.2.

Remark 2.7 Let $u \in \mathscr{K}$ be invertible. Then $f \mapsto f \otimes \mathrm{id}_{u}$ induces an isomorphism $\operatorname{Hom}_{\mathscr{K}}(a, b) \stackrel{\sim}{\rightarrow} \operatorname{Hom}_{\mathscr{K}}(a \otimes u, b \otimes u)$ for all $a, b \in \mathscr{K}$. In particular, there exists a central unit $\epsilon_{u} \in \mathrm{R}_{\mathscr{K}}^{\times}$such that the switch $\sigma_{u, u}: u \otimes u \stackrel{\sim}{\rightarrow} u \otimes u$ is equal to $\left.\left(\epsilon_{u}\right)\right|_{u \otimes u}=\epsilon_{u} \cdot \operatorname{id}_{u} \otimes u$. Note that $\left(\sigma_{u, u}\right)^{2}=\operatorname{id}_{u \otimes u}$ implies $\left(\epsilon_{u}\right)^{2}=1$.

Definition 2.8 We say that an object $x \in \mathscr{K}$ has central switch if there exists a unit $\epsilon=\epsilon(x) \in \mathrm{R}_{\mathscr{K}}^{\times}$such that $\sigma_{x, x}=\epsilon_{x} \otimes x: x \otimes x \rightarrow x \otimes x$. Remark 2.7 shows that invertible objects have central switch.

Remark 2.9 Assume that $x$ and $y$ have central switch. Then $x \otimes y$ also does. Indeed, for $x_{1}, x_{2}, y_{1}, y_{2} \in \mathscr{K}$ the switch $\sigma_{x_{1} \otimes y_{1}, x_{2} \otimes y_{2}}$ decomposes as follows:

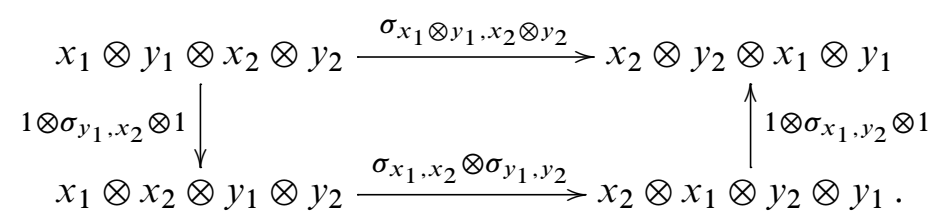


For $x_{1}=x_{2}=x$ and $y_{1}=y_{2}=y$, the bottom morphism is central $(=\epsilon(x) \cdot \epsilon(y))$ and the two vertical morphisms are mutual inverses. So $x \otimes y$ has central switch: $\epsilon(x \otimes y)=\epsilon(x) \cdot \epsilon(y)$ in $\mathrm{R}_{\mathscr{C}}$. In particular, $x^{\otimes n}$ has central switch for all $n \geq 1$.

Lemma 2.10 Let $f: a \rightarrow x$ and $g: b \rightarrow x$ be two morphisms and assume that $x$ has central switch (Definition 2.8). Then there exists an isomorphism $\tau: b \otimes a \stackrel{\sim}{\rightarrow} a \otimes b$ such that $g \otimes f=(f \otimes g) \circ \tau$. Similarly, for $f^{\prime}: x \rightarrow a^{\prime}$ and $g^{\prime}: x \rightarrow b^{\prime}$ there exists an isomorphism $\tau^{\prime}: a^{\prime} \otimes b^{\prime} \stackrel{\sim}{\rightarrow} b^{\prime} \otimes a^{\prime}$ such that $g^{\prime} \otimes f^{\prime}=\tau^{\prime} \circ\left(f^{\prime} \otimes g^{\prime}\right)$.

Proof This is elementary. Let $\epsilon \in \mathrm{R}_{\mathscr{K}}^{\times}$such that $\sigma_{x, x}=\left.\epsilon\right|_{x \otimes x}$. Since the diagram

$$
\begin{aligned}
b \otimes a \stackrel{g \otimes f}{\longrightarrow} & x \otimes x \\
\sigma_{b, a} \downarrow \cong \quad & \cong \downarrow \sigma_{x, x}=\left.\epsilon\right|_{x \otimes x} \\
a \otimes b \stackrel{f \otimes g}{\longrightarrow} & x \otimes x
\end{aligned}
$$

commutes, and since $\epsilon \in \mathrm{R}_{\mathscr{K}}$ is "central" (Proposition 2.2), we can take $\tau=\epsilon \cdot \sigma_{b, a}$.

Lemma 2.11 Let $g: x \rightarrow y$ be a morphism. Let

$$
a \stackrel{k}{\longrightarrow} b \stackrel{l}{\longrightarrow} c \stackrel{m}{\longrightarrow} \Sigma a
$$

be a distinguished triangle and suppose that the following diagram commutes:

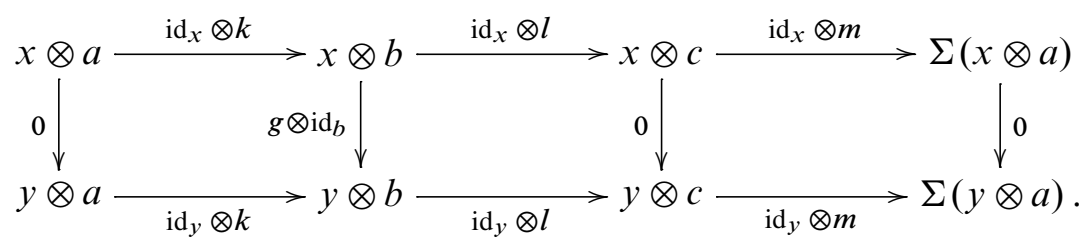

Then the morphism $g \otimes g \otimes \mathrm{id}_{b}: x \otimes x \otimes b \longrightarrow y \otimes y \otimes b$ is equal to zero.

Proof From the commutative diagram, we get

(a) $\left(g \otimes \mathrm{id}_{b}\right) \circ\left(\mathrm{id}_{x} \otimes k\right)=0$

(b) $g \otimes l=\left(\mathrm{id}_{y} \otimes l\right) \circ\left(g \otimes \mathrm{id}_{b}\right)=0$.

By (a) and the exact triangle of the first row, there exists $h: x \otimes c \rightarrow y \otimes b$ such that $g \otimes \mathrm{id}_{b}=h \circ\left(\mathrm{id}_{x} \otimes l\right)$. Hence $g \otimes g \otimes \mathrm{id}_{b}=g \otimes\left(h \circ\left(\mathrm{id}_{x} \otimes l\right)\right)=\left(\mathrm{id}_{y} \otimes h\right) \circ\left(g \otimes \mathrm{id}_{x} \otimes l\right)$ and this is zero because $g \otimes \mathrm{id}_{x} \otimes l=0$. To check this last claim, note that (b) implies $0=\mathrm{id}_{x} \otimes g \otimes l: x \otimes x \otimes b \longrightarrow x \otimes y \otimes c$ and switch the first two factors, that is, precompose by $\sigma_{x, x} \otimes \mathrm{id}_{b}$ and postcompose by $\sigma_{x, y} \otimes \mathrm{id}_{c}$. 
Proposition 2.12 Let $f: x \rightarrow y$ be a morphism. Then the objects $a \in \mathscr{K}$ for which there exists $n \geq 1$ with $f^{\otimes n} \otimes \mathrm{id}_{a}=0$ (as a morphism $x^{\otimes n} \otimes a \longrightarrow y^{\otimes n} \otimes a$ ) form a thick triangulated $\otimes$-ideal of $\mathscr{K}$.

Proof Thick and $\otimes$-ideal is easy; triangulated follows from Lemma 2.11.

The following is well-known for $x=y=\mathbb{1}$.

Proposition 2.13 Let $f: x \rightarrow y$ be a morphism and assume that $x$ and $y$ have central switch (Definition 2.8); eg $x$ and $y$ invertible. Then $(f \otimes f) \otimes \operatorname{id}_{\text {cone }(f)}=0$.

Proof Consider a distinguished triangle

$$
x \stackrel{f}{\longrightarrow} y \stackrel{f_{1}}{\longrightarrow} \operatorname{cone}(f) \stackrel{f_{2}}{\longrightarrow} \Sigma(x)
$$

and the commutative diagram

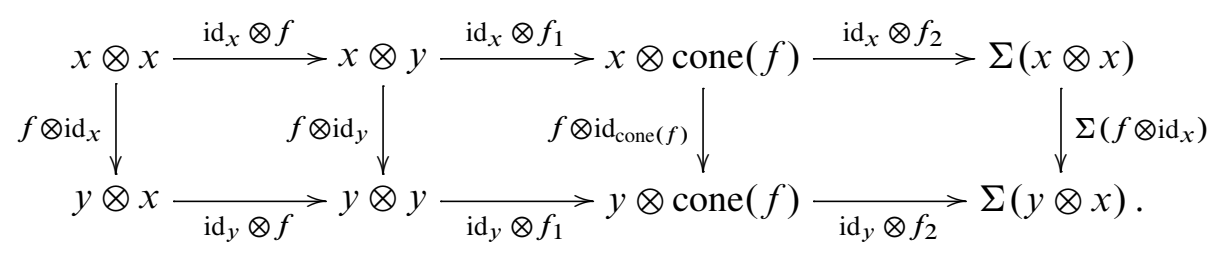

We claim that the diagonal morphisms of the second and third squares vanish:

(a) $\left(\operatorname{id}_{y} \otimes f_{1}\right) \circ\left(f \otimes \operatorname{id}_{y}\right)=0$.

(b) $\Sigma\left(f \otimes \mathrm{id}_{x}\right) \circ\left(\mathrm{id}_{x} \otimes f_{2}\right)=0$.

Since $y$ has central switch, we can apply Lemma 2.10 to our $f$ and to $g:=\mathrm{id}_{y}$ to obtain an isomorphism $\tau: y \otimes x \stackrel{\sim}{\rightarrow} x \otimes y$ such that $\left(f \otimes \mathrm{id}_{y}\right) \circ \tau=\mathrm{id}_{y} \otimes f$. Then (a) is easily checked by composing on the right by the isomorphism $\tau$ and using that $f_{1} \circ f=0$. Similarly, for (b), using the second statement of Lemma 2.10.

By (a) and (b), we can now replace the above morphism of distinguished triangles $\left(f \otimes \mathrm{id}_{x}, f \otimes \mathrm{id}_{y}, f \otimes \mathrm{id}_{\text {cone }(f)}\right)$ by the morphism $\left(0,0, f \otimes \operatorname{id}_{\operatorname{cone}(f)}\right)$. The result then follows from Lemma 2.11 (modulo obvious rotations).

Proposition 2.14 Let $f: x \rightarrow y$ be a morphism in $\mathscr{K}$ and suppose that $\langle x, y\rangle=\mathscr{K}$, eg $x$ or $y$ is invertible. If $a \in \mathscr{K}$ is such that $f \otimes \operatorname{id}_{a}=0$ then $a \in\langle\operatorname{cone}(f)\rangle$. 
Proof Tensor the distinguished triangle

$$
x \stackrel{f}{\longrightarrow} y \rightarrow \operatorname{cone}(f) \rightarrow \Sigma(x)
$$

with $a$ and use the assumption $f \otimes \mathrm{id}_{a}=0$ to deduce that $(y \otimes a) \oplus \Sigma(x \otimes a) \simeq$ cone $(f) \otimes a$. This shows that $x \otimes a$ and $y \otimes a$ belong to $\langle$ cone $(f)\rangle$. Therefore $\langle x \otimes a, y \otimes a\rangle \subset\langle\operatorname{cone}(f)\rangle$. Finally, from $\mathbb{1} \in\langle x, y\rangle$ we deduce $a \in\langle x \otimes a, y \otimes a\rangle$ by Proposition 1.7 (a).

Theorem 2.15 Assume that $x, y \in \mathscr{K}$ have central switch (Definition 2.8) and that $\langle x, y\rangle=\mathscr{K}$; eg $x$ and $y$ invertible. Let $f: x \rightarrow y$ be a morphism in $\mathscr{K}$. Then the thick triangulated $\otimes$-ideal generated by its cone, $\langle$ cone $(f)\rangle$, coincides with the subcategory of those objects $a \in \mathscr{K}$ for which $f^{\otimes n} \otimes \mathrm{id}_{a}=0$ for some $n \geq 1$.

Proof Let $\mathscr{F}:=\left\{a \in \mathscr{K} \mid \exists n \geq 1\right.$ such that $\left.f^{\otimes n} \otimes \mathrm{id}_{a}=0\right\}$ the subcategory in question. By Proposition 2.12, $\mathscr{F}$ is a thick triangulated $\otimes$-ideal and by Proposition 2.13 we have cone $(f) \in \mathscr{F}$, hence $\langle\operatorname{cone}(f)\rangle \subset \mathscr{F}$. Let us now check the other inclusion.

Let $a \in \mathscr{K}$ be such that $f^{\otimes n} \otimes \mathrm{id}_{a}=0$. Then $a \in\left\langle\operatorname{cone}\left(f^{\otimes n}\right)\right\rangle$ by Proposition 2.14 applied to $f^{\otimes n}: x^{\otimes n} \rightarrow y^{\otimes n}$. Note that $\left\langle x^{\otimes n}, y^{\otimes n}\right\rangle=\mathscr{K}$ by Proposition 1.7 (b).

So, it remains to prove that $\left\langle\operatorname{cone}\left(f^{\otimes n}\right)\right\rangle \subset\langle\operatorname{cone}(f)\rangle$ for all $n \geq 1$. We do so by induction on $n$. Suppose that $n \geq 1$ and that $\left\langle\operatorname{cone}\left(f^{\otimes n}\right)\right\rangle \subset\langle\operatorname{cone}(f)\rangle$. Then the octahedron axiom applied to the relation $f^{\otimes(n+1)}=\left(f \otimes \mathrm{id}_{y} \otimes n\right) \circ\left(\mathrm{id}_{x} \otimes f^{\otimes n}\right)$ shows that cone $\left(f^{\otimes(n+1)}\right) \in\left\langle\operatorname{cone}(f)\right.$, cone $\left.\left(f^{\otimes n}\right)\right\rangle \subset\langle\operatorname{cone}(f)\rangle$, which gives the result.

Proposition 2.16 Let $x, y \in \mathscr{K}$ be as in Theorem 2.15, eg $x$ and $y$ invertible. Let $f: x \rightarrow y$ and $n \geq 1$. Then $\left\langle\operatorname{cone}(f)^{\otimes n}\right\rangle=\left\langle\operatorname{cone}\left(f^{\otimes n}\right)\right\rangle=\langle\operatorname{cone}(f)\rangle$.

Proof Note that $x^{\otimes n}$ and $y^{\otimes n}$ still satisfy the hypotheses of Theorem 2.15 by Remark 2.9 and Proposition 1.7 (b). It is then direct from Theorem 2.15 that $\langle$ cone $(f)\rangle=$ $\left\langle\right.$ cone $\left.\left(f^{\otimes n}\right)\right\rangle$. Obviously, cone $(f)^{\otimes n} \in\langle\operatorname{cone}(f)\rangle$ hence it suffices to prove that cone $(f) \in\left\langle\operatorname{cone}(f)^{\otimes n}\right\rangle$. By induction (and Proposition 1.7 (a)), it suffices to treat the case $n=2$. Consider the composition

$x \otimes x \otimes \operatorname{cone}(f) \stackrel{f \otimes 1 \otimes 1}{\longrightarrow} y \otimes x \otimes \operatorname{cone}(f) \stackrel{1 \otimes f \otimes 1}{\longrightarrow} y \otimes y \otimes \operatorname{cone}(f)$.

Since the cone of the first map, $\operatorname{cone}(f) \otimes x \otimes \operatorname{cone}(f)$, and the cone of the second, $y \otimes \operatorname{cone}(f) \otimes \operatorname{cone}(f)$, both belong to $\left\langle\operatorname{cone}(f)^{\otimes 2}\right\rangle$, the octahedron axiom tells us that the cone of the composition also belongs to $\left\langle\operatorname{cone}(f)^{\otimes 2}\right\rangle$. But that composition 
is zero by Proposition 2.13 hence its cone is $\Sigma\left(x^{\otimes 2} \otimes \operatorname{cone}(f)\right) \oplus\left(y^{\otimes 2} \otimes \operatorname{cone}(f)\right)$. By definition, $\left\langle\right.$ cone $\left.(f)^{\otimes 2}\right\rangle$ is thick, so we have proved

$$
\left\langle x^{\otimes 2} \otimes \operatorname{cone}(f), y^{\otimes 2} \otimes \operatorname{cone}(f)\right\rangle \subset\left\langle\operatorname{cone}(f)^{\otimes 2}\right\rangle .
$$

Proposition 1.7 (b) and (a) gives us $\mathbb{1} \in\left\langle x^{\otimes 2}, y^{\otimes 2}\right\rangle$ and cone $(f)=\mathbb{1} \otimes \operatorname{cone}(f) \in$ $\left\langle x^{\otimes 2} \otimes \operatorname{cone}(f), y^{\otimes 2} \otimes \operatorname{cone}(f)\right\rangle \subset\left\langle\right.$ cone $\left.(f)^{\otimes 2}\right\rangle$, as wanted.

\section{Graded homomorphisms and central localization}

Notation 3.1 Let us fix $u \in \mathscr{K}$ an invertible object. For any pair of objects $a, b \in \mathscr{K}$, and any $i \in \mathbb{Z}$, we denote by

$$
\operatorname{Hom}_{\mathscr{K}}^{i}(a, b):=\operatorname{Hom}_{\mathscr{K}}\left(a, u^{\otimes i} \otimes b\right) .
$$

When we need to stress the dependency on $u$, we can write $\operatorname{Hom}_{\mathscr{K}, u}^{i}(a, b)$. The above notation is commonly used with $u=\Sigma(\mathbb{1})$, for which $\operatorname{Hom}_{\mathscr{K}}^{i}(a, b) \cong \operatorname{Hom}_{\mathscr{K}}\left(a, \Sigma^{i}(b)\right)$. We denote by $\operatorname{Hom}_{\mathscr{K}}^{\bullet}(a, b)$ the $\mathbb{Z}$-graded abelian group $\bigoplus_{i \in \mathbb{Z}} \operatorname{Hom}_{\mathscr{K}}^{i}(a, b)$. We have an obvious composition:

$$
\begin{array}{ccc}
\operatorname{Hom}_{\mathscr{K}}^{j}(b, c) \times \operatorname{Hom}_{\mathscr{K}}^{i}(a, b) & \stackrel{\diamond}{\longrightarrow} & \operatorname{Hom}_{\mathscr{K}}^{i+j}(a, c) \\
\left(b \stackrel{g}{\longrightarrow} u^{\otimes j} \otimes c, a \stackrel{f}{\longrightarrow} u^{\otimes i} \otimes b\right) & \longmapsto & a \underbrace{\overbrace{\operatorname{id}_{u}^{\otimes i} \otimes g}^{\otimes}}_{u^{\otimes i} \otimes b} u^{\otimes(i+j)} \otimes c
\end{array}
$$

which coincides with the usual composition when $i=0$.

We shall mostly use the case $u=\Sigma(\mathbb{1})$ but the above flexibility can be useful in general, as illustrated in algebraic geometry in Remark 8.2.

Definition 3.2 We denote by $\mathrm{R}_{\mathscr{K}}^{\bullet}$ (or $\mathrm{R}_{\mathscr{K}, u}^{\bullet}$ ) the graded group $\operatorname{Hom}_{\mathscr{K}}^{\bullet}(\mathbb{1}, \mathbb{1}$ ), which is a unital associative $\mathbb{Z}$-graded ring with respect to $\diamond$. We call it the graded central ring of $\mathscr{K}$ (with respect to $u \in \mathscr{K}$ ). Note that $\mathrm{R}_{\mathscr{K}}^{0}$ is just the central ring $\mathrm{R}_{\mathscr{K}}=\operatorname{End}_{\mathscr{K}}(\mathbb{1})$ of Section 2, independently of $u$.

Generalizing Proposition 2.2, we have the following compatibility between $\diamond$ and $\otimes$. Recall the central unit $\epsilon_{u} \in \mathrm{R}_{\mathscr{K}}=\mathrm{R}_{\mathscr{K}}^{0}$ such that the switch $\sigma_{u, u}: u \otimes u \stackrel{\sim}{\rightarrow} u \otimes u$ is given by multiplication by $\epsilon_{u}$. (See Remark 2.7.) Of course, we have $\left(\epsilon_{u}\right)^{2}=1$ and the reader can simply assume that $\epsilon_{u}= \pm 1$, as it is often the case. 
Proposition 3.3 Let $u \in \mathscr{K}$ be an invertible object. With the above notation:

(a) For $a, b \in \mathscr{K}$, the graded abelian group $\operatorname{Hom}_{\mathscr{K}}^{\bullet}(a, b)$ carries both a structure of left and of right graded module over the graded central ring $\mathrm{R}_{\mathscr{\kappa}}^{\bullet}$, defined via the tensor product $\otimes$. Explicitly, for $f \in \mathrm{R}_{\mathscr{K}}^{i}$ and $g \in \operatorname{Hom}_{\mathscr{K}}^{j}(a, b)$, we set

$$
\begin{aligned}
& f \cdot g:=\left(a \stackrel{f \otimes g}{\longrightarrow} u^{\otimes i} \otimes u^{\otimes j} \otimes b \cong u^{\otimes(i+j)} \otimes b\right), \\
& g \cdot f:=\left(a \stackrel{g \otimes f}{\longrightarrow} u^{\otimes j} \otimes b \otimes u^{\otimes i} \stackrel{\text { id } \otimes \sigma}{\cong} u^{\otimes j} \otimes u^{\otimes i} \otimes b \cong u^{\otimes(i+j)} \otimes b\right) .
\end{aligned}
$$

(b) When $a=b=\mathbb{1}$, both actions coincide with composition $f \cdot g=f \diamond g$.

(c) Composition $\diamond$ : $\operatorname{Hom}_{\mathscr{K}}^{\bullet}(b, c) \times \operatorname{Hom}_{\mathscr{K}}^{\bullet}(a, b) \longrightarrow \operatorname{Hom}_{\mathscr{K}}^{\bullet}(a, c)$ is bilinear with respect to both the left action and the right action, in an $\epsilon_{u}$-graded way, that is, for $f \in \mathrm{R}_{\mathscr{Y}}^{i}, g \in \operatorname{Hom}_{\mathscr{K}}^{j}(a, b)$ and $h \in \operatorname{Hom}_{\mathscr{K}}^{k}(b, c)$, we have

$$
\begin{aligned}
& (f \cdot h) \diamond g=f \cdot(h \diamond g)=\left(\epsilon_{u}\right)^{i \cdot k} h \diamond(f \cdot g), \\
& h \diamond(g \cdot f)=(h \diamond g) \cdot f=\left(\epsilon_{u}\right)^{i \cdot j}(h \cdot f) \diamond g .
\end{aligned}
$$

(d) The left and right actions coincide in an $\epsilon_{u}$-graded way, that is, for $f \in \mathrm{R}_{\mathscr{K}}^{i}$ and $g \in \operatorname{Hom}_{\mathscr{K}}^{j}(a, b)$, we have

$$
f \cdot g=\left(\epsilon_{u}\right)^{i \cdot j} g \cdot f .
$$

(e) In particular, the $\mathbb{Z}$-graded central ring $\mathrm{R}_{\mathscr{K}}^{\bullet}$ is $\epsilon_{u}$-commutative.

Sketch of Proof Consider the external product

$$
\operatorname{Hom}_{\mathscr{K}}^{i}(a, b) \times \operatorname{Hom}_{\mathscr{K}}^{j}(c, d) \stackrel{\otimes}{\longrightarrow} \operatorname{Hom}_{\mathscr{K}}^{i+j}(a \otimes c, b \otimes d)
$$

defined for $f \in \operatorname{Hom}_{\mathscr{K}}^{i}(a, b)$ and $g \in \operatorname{Hom}_{\mathscr{K}}^{j}(c, d)$ by the formula

$f \otimes g$

$a \otimes c \underset{f \otimes g}{\vec{\longrightarrow}} u^{\otimes i} \otimes b \otimes u^{\otimes j} \otimes d \underset{1 \otimes \sigma \otimes 1}{\longrightarrow} u^{\otimes i} \otimes u^{\otimes j} \otimes b \otimes d=u^{\otimes i+j} \otimes b \otimes d$.

This product is associative, natural and $\epsilon_{u}$-commutative, in the obvious sense. Statements (a)-(e) can be deduced from these properties. (Compare Proposition 2.2.) Further verifications are left to the reader, who can find some details in [11, Section 2.1.2].

Remark 3.4 We are going to need some elementary graded commutative algebra over $\epsilon$-commutative $\mathbb{Z}$-graded rings. Let $R^{\bullet}=\bigoplus_{i \in \mathbb{Z}} R^{i}$ be a unitary and associative 
$\mathbb{Z}$-graded ring. We denote by $R^{\text {hom }}:=\bigcup_{i \in \mathbb{Z}} R^{i}$ the subset of homogeneous elements. We say that $R^{\bullet}$ is $\epsilon$-commutative if there exists $\epsilon \in R^{0}$ such that $\epsilon^{2}=1$ and

$$
f \cdot g=\epsilon^{i j} \cdot g \cdot f
$$

for all homogeneous $f \in R^{i}$ and $g \in R^{j}$. Hence the subset $R^{\text {even }}:=\bigcup_{i \in \mathbb{Z}} R^{2 i}$ is central in $R^{\bullet}$, and in particular so is the unit $\epsilon$.

Let $S \subset R^{\text {hom }}$ be a central multiplicative subset $(1 \in S$ and $S \cdot S \subset S$, typically $\left.S \subset R^{\text {even }}\right)$. Then the localization $S^{-1} R^{\bullet}$ is graded as usual by setting $\left(S^{-1} R^{\bullet}\right)^{i}:=$ $\left\{f / s \mid f \in R^{i+j}, s \in S \cap R^{j}\right\}$. If we need to invert a more general multiplicative subset $T \subset R^{\text {hom }}$, hypothesis (3-1) allows us to do it by inverting the central subset $S:=\left\{t^{2} \mid t \in T\right\}$ instead.

We define the homogeneous spectrum, $\operatorname{Spec}^{\mathrm{h}}\left(R^{\bullet}\right)$, as the set of proper homogeneous ideals $\mathfrak{p}^{\bullet}$ of $R^{\bullet}$ which are prime $\left(a \cdot b \in \mathfrak{p}^{\bullet}\right.$ implies $a \in \mathfrak{p}^{\bullet}$ or $b \in \mathfrak{p}^{\bullet}$; this can be checked on homogeneous elements only). The Zariski topology has as closed subsets the $V\left(I^{\bullet}\right):=\left\{\mathfrak{p}^{\bullet} \in \operatorname{Spec}^{\mathrm{h}}\left(R^{\bullet}\right) \mid I^{\bullet} \subset \mathfrak{p}^{\bullet}\right\}$ for homogeneous ideals $I^{\bullet} \subset R^{\bullet}$. The principal open subsets $D(s):=\left\{\mathfrak{p}^{\bullet} \in \operatorname{Spec}^{\mathrm{h}}\left(R^{\bullet}\right) \mid s \notin \mathfrak{p}^{\bullet}\right\}$ define a basis of the topology, for $s$ running through $R^{\text {hom }}$, or just through $R^{\text {even }}$ since $D(s)=D\left(s^{2}\right)$. We define a structure sheaf $\mathcal{O}^{\bullet}$ of $\epsilon$-commutative $\mathbb{Z}$-graded rings on $\operatorname{Spec}^{\mathrm{h}}\left(R^{\bullet}\right)$ by setting $\mathcal{O}^{\bullet}(D(s))=R^{\bullet}[1 / s]:=S^{-1} R^{\bullet}$ where $S=\left\{s^{2 i} \mid i \in \mathbb{N}\right\} \subset R^{\text {even }}$.

When $\epsilon=1$, the reader can find in Lorenzini [20] all results we use below. We also use this reference even for $\epsilon \neq 1$. Unfortunately, the literature does not seem to have an $\epsilon$-commutative equivalent of [20] and we will follow here the usual practice of leaving to the reader to extend the proofs in the $\epsilon$-commutative case.

Here, we need to associate to a homogeneous prime $\mathfrak{p}^{\bullet} \subset R^{\bullet}$ a localization $R^{\bullet} \rightarrow$ $S^{-1} R^{\bullet}$ such that $S^{-1} R^{\bullet}$ has a unique maximal homogeneous ideal (ie $S^{-1} R^{\bullet}$ is a local graded ring) in such a way that this maximal ideal maps to our $\mathfrak{p}^{\bullet}$ under the natural map (defined as usual)

$$
\operatorname{Spec}^{\mathrm{h}}\left(S^{-1} R^{\bullet}\right) \hookrightarrow \operatorname{Spec}^{\mathrm{h}}\left(R^{\bullet}\right) .
$$

This can be realized by localization with respect to the central multiplicative subset

$$
S_{\mathfrak{p} \bullet}:=\left\{s \in R^{\text {even }} \text { such that } s \notin \mathfrak{p}^{\bullet}\right\} \text {. }
$$

Indeed, a homogeneous prime ideal $\mathfrak{q}^{\bullet} \subset R^{\bullet}$ disjoint from $S_{\mathfrak{p}}$ is contained in $\mathfrak{p}^{\bullet}$ (otherwise, there is a homogeneous $t \in \mathfrak{q}^{\bullet}$ with $t \notin \mathfrak{p}^{\bullet}$, but then $t^{2} \in S_{\mathfrak{p}} \bullet \cap \mathfrak{q}^{\bullet}=\varnothing$ ).

Construction 3.5 Let us return to our tensor triangulated category $\mathscr{K}$. Consider now $S \subset \mathrm{R}_{\mathscr{K}}^{\bullet}$ a multiplicative subset of homogeneous elements $(1 \in S$ and $S \cdot S \subset S)$. 
When $\epsilon_{u} \neq 1$, we further require $S \subset \mathrm{R}_{\mathscr{K}}^{\text {even }}$. So, $S$ is central in $\mathrm{R}_{\mathscr{K}}^{\bullet}$ and acts centrally on any graded module $\operatorname{Hom}_{\mathscr{K}}^{\bullet}(a, b)$. For instance, for each $\mathfrak{p}^{\bullet} \in \operatorname{Spec}^{\mathrm{h}}\left(\mathrm{R}_{\mathscr{K}}^{\bullet}\right)$, we can take $S=S_{\mathfrak{p}} \bullet$ as defined in (3-2) above.

We can construct as above the $\mathbb{Z}$-graded $\epsilon_{u}$-commutative ring $S^{-1} \mathrm{R}_{\mathscr{K}}^{\bullet}$. Then, for any $a, b \in \mathscr{K}$, we can construct the $S^{-1} \mathrm{R}_{\mathscr{K}}^{\bullet}$-module $S^{-1} \operatorname{Hom}_{\mathscr{K}}^{\bullet}(a, b)$. Let us denote by $\left(S^{-1} \operatorname{Hom}_{\mathscr{K}}^{\bullet}(a, b)\right)^{0}$ its degree zero part. Composition of morphisms $(\diamond)$ being $\mathrm{R}_{\mathscr{K}}^{\bullet}$-bilinear, it induces a well-defined homomorphism

$$
\left(S^{-1} \operatorname{Hom}_{\mathscr{K}}^{\bullet}(b, c)\right)^{0} \times\left(S^{-1} \operatorname{Hom}_{\mathscr{K}}^{\bullet}(a, b)\right)^{0} \longrightarrow\left(S^{-1} \operatorname{Hom}_{\mathscr{K}}^{\bullet}(a, c)\right)^{0} .
$$

This defines a new category, that we denote $S^{-1} \mathscr{K}$, with the same objects as $\mathscr{K}$ and

$$
\operatorname{Hom}_{S^{-1 \mathscr{K}}}(a, b)=\left(S^{-1} \operatorname{Hom}_{\mathscr{K}}^{\bullet}(a, b)\right)^{0} .
$$

The natural homomorphism $\operatorname{Hom}_{\mathscr{K}}(a, b) \longrightarrow\left(S^{-1} \operatorname{Hom}_{\mathscr{K}}^{\bullet}(a, b)\right)^{0}$ defines a functor

$$
q_{S}: \mathscr{K} \longrightarrow S^{-1} \mathscr{K} .
$$

We now show that $S^{-1} \mathscr{K}$ is a Verdier localization of $\mathscr{K}$, in the obvious way. (Compare Hovey et al [16, Theorem 3.3.7] under additional hypotheses about $\mathscr{K}$.)

Theorem 3.6 Let us keep notation as above. Consider $\mathscr{g}=\langle\operatorname{cone}(s) \mid s \in S\rangle$ the thick triangulated $\otimes$-ideal generated by the cones of elements of $S$. Consider the Verdier localization $q: \mathscr{K} \rightarrow \mathscr{K} / \mathscr{F}$. Then there is an equivalence $\alpha: S^{-1} \mathscr{K} \stackrel{\sim}{\rightarrow} \mathscr{K} / \mathscr{F}$ such that $\alpha \circ q_{S}=q$. In particular, $S^{-1} \mathscr{K}$ can be equipped with the structure of a tensor triangulated category such that $q_{S}: \mathscr{K} \longrightarrow S^{-1} \mathscr{K}$ is a morphism of tensor triangulated categories.

We need two preliminary results. The first one is a generalization of Theorem 2.15. Recall from Notation 2.6 the shorthand $f t_{a}=f \otimes \mathrm{id}_{a}$ for $f \in \mathrm{R}_{\mathscr{K}}^{\text {even }}$ and an object $a \in \mathscr{K}$.

Proposition 3.7 With notation as in Construction 3.5 and Theorem 3.6, we have $\mathscr{g}=\left\{c \in \mathscr{K} \mid \exists s \in S\right.$ such that $\left.s \downarrow_{c}=0\right\}$.

Proof Let $\mathscr{I}^{\prime}:=\left\{c \in \mathscr{K} \mid \exists s \in S\right.$ such that $\left.s \downarrow_{c}=0\right\}$. Proposition 2.14 implies $\mathscr{F}^{\prime} \subset \mathscr{F}$. To check the converse, first note that since $S$ is multiplicative and since multiplication and composition coincide on $\mathrm{R}_{\mathscr{K}}^{\bullet}$, we have $\mathscr{g}^{\prime}=\{c \in \mathscr{K} \mid \exists s \in$ $S$ and $n \geq 1$ such that $\left.\left(s^{\otimes n}\right) \otimes \mathrm{id}_{c}=0\right\}$. It is then easy to deduce from Proposition 2.12 that $\mathscr{g}^{\prime}$ is thick, triangulated and $\otimes$-ideal. By Proposition 2.13, we have cone $(s) \in \mathscr{g}^{\prime}$ for all $s \in S$. Therefore $\mathscr{g}=\langle\operatorname{cone}(s) \mid s \in S\rangle \subset \mathscr{g}^{\prime}$. 
Lemma 3.8 With notation as in Construction 3.5 and Theorem 3.6, a morphism $k: b \rightarrow x$ in $\mathscr{K}$ has its cone in $\mathscr{F}$ if and only if there exists $s \in S$ of some degree $d \in \mathbb{Z}$ and $l, m \in \operatorname{Hom}_{\mathscr{K}}^{d}(x, b)$ such that $l \circ k=s l_{b}$ in $\operatorname{Hom}_{\mathscr{K}}^{d}(b, b)$ and $k \diamond m=s l_{x}$ in $\operatorname{Hom}_{\mathscr{K}}^{d}(x, x)$.

Proof Consider a distinguished triangle over $k: b \rightarrow x$ and, for any $s \in S$ of degree $d$, the commutative (plain) diagram:

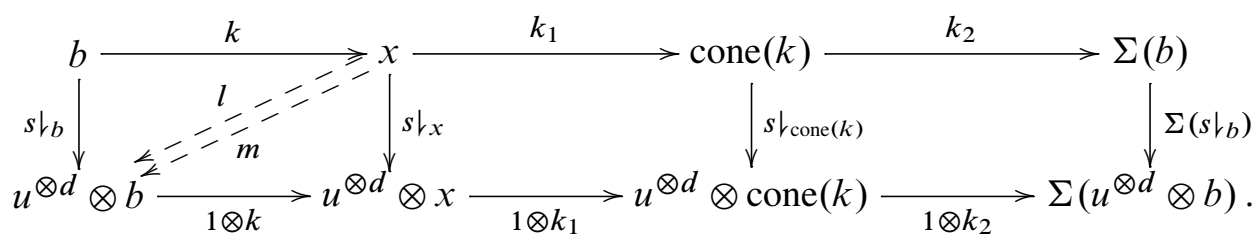

If cone $(k) \in \mathscr{E}$, by Proposition 3.7, we can choose $s \in S$ such that the third vertical morphism $s l_{\text {cone }(k)}$ vanishes. Then $\left(1 \otimes k_{1}\right) \circ s l_{x}=0$ and $s \downarrow_{b} \circ \Sigma^{-1}\left(k_{2}\right)=0$ and the existence of $l$ and $m$ with $l \circ k=\left.s\right|_{b}$ and $(1 \otimes k) \circ m=s \downarrow_{x}$ are standard facts in triangulated categories. Conversely, if such morphisms $l$ and $m$ exist for some $s \in S$, then $s \downarrow_{\text {cone }(k)} \circ k_{1}=0$ and $\left(1 \otimes k_{2}\right) \circ s l_{\text {cone }(k)}=0$. By Lemma 2.11 , this implies $\left(s^{2}\right) \downarrow_{\text {cone }(k)}=s \otimes s \otimes \operatorname{id}_{\text {cone }(k)}=0$. Since $s^{2} \in S$ as well, we have just proved cone $(k) \in \mathscr{g}$ by Proposition 3.7.

Proof of Theorem 3.6 We define the functor $\alpha: S^{-1} \mathscr{K} \rightarrow \mathscr{K} / \mathscr{F}$ as the identity on objects. Let $a, b \in \mathscr{K}$. For any $s \in S$, we have cone $\left(s \downarrow_{b}\right)=\operatorname{cone}(s) \otimes b \in \mathscr{J}$. Therefore $q\left(s l_{b}\right)$ is an isomorphism in $\mathscr{K} / \mathscr{f}$ and we have a natural homomorphism

$$
\begin{aligned}
\alpha_{a, b}:\left(S^{-1} \operatorname{Hom}_{\mathscr{K}}^{\bullet}(a, b)\right)^{0} & \longrightarrow \operatorname{Hom}_{\mathscr{K} / \mathscr{g}}(a, b) \\
f / s & \longmapsto q\left(s \iota_{b}\right)^{-1} \circ f .
\end{aligned}
$$

It is easy to deduce from Lemma 3.8 that $\alpha_{a, b}$ is an isomorphism of abelian groups. Indeed, Lemma 3.8 shows that any fraction in $\mathscr{K} / \mathscr{F}$ can be amplified to have denominator in $S$. Explicitly, a morphism $a \rightarrow b$ in $\mathscr{K} / \mathscr{F}$ can be represented by a fraction $k^{-1} g$ as in the upper row of the following diagram:

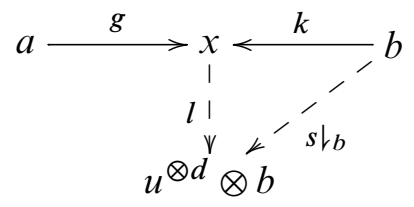

with cone $(k) \in \mathscr{F}$. Lemma 3.8 gives us a morphism $l \in \operatorname{Hom}_{\mathscr{K}}^{d}(x, b)$ such that $l \circ k=s \iota_{b}$ for some $s \in S$. Then the morphism $k^{-1} g$ is equal to $\alpha_{a, b}(\mathrm{lg} / \mathrm{s})$. Injectivity of $\alpha_{a, b}$ follows from Lemma 3.8 in a similar way. 
Corollary 3.9 Let $\mathfrak{p}^{\bullet} \in \operatorname{Spec}^{\mathrm{h}}\left(\mathrm{R}_{\mathscr{K}}^{\bullet}\right)$. The localization $\mathscr{K}_{\mathfrak{p}} \bullet:=S_{\mathfrak{p}^{\bullet}}^{-1} \mathscr{K}$ where $S_{\mathfrak{p}} \bullet=$ $\left\{s \in \mathrm{R}_{\mathscr{K}}^{\text {even }} \mid s \notin \mathfrak{p}^{\bullet}\right\}$ has graded central ring $\operatorname{End}_{\mathscr{K}_{\mathfrak{p}} \bullet}^{\bullet}(\mathbb{1})$ isomorphic to $\left(\mathrm{R}_{\mathscr{K}}^{\bullet}\right)_{\mathfrak{p}} \bullet$

Corollary 3.10 Let $S \subset$ End $_{\mathscr{K}}(\mathbb{1})$ be a multiplicative subset of the central ring $\mathrm{R}_{\mathscr{K}}$. Then the Verdier quotient $S^{-1} \mathscr{K}$ of $\mathscr{K}$ by the thick triangulated $\otimes$-ideal $\langle\operatorname{cone}(s)|$ $s \in S\rangle$ has the same objects as $\mathscr{K}$ and morphisms $\operatorname{Hom}_{S^{-1} \mathscr{K}}(a, b)=S^{-1} \operatorname{Hom}_{\mathscr{K}}(a, b)$.

Proof This can be checked as above or deduced from Theorem 3.6 (using any invertible object $u$ ), using that for a graded $R^{\bullet}$-module $M^{\bullet}$ and for $S \subset R^{0}$, we have $\left(S^{-1} M^{\bullet}\right)^{0}=S^{-1}\left(M^{0}\right)$.

Corollary 3.11 Let $\mathfrak{p} \in \operatorname{Spec}\left(\mathrm{R}_{\mathscr{K}}\right)$. Then the localization $\mathscr{K}_{\mathfrak{p}}:=S_{\mathfrak{p}}^{-1} \mathscr{K}$ where $S_{\mathfrak{p}}:=$ $\mathrm{R}_{\mathscr{K}} \backslash \mathfrak{p}$ has central ring $\operatorname{End}_{\mathscr{K}_{\mathfrak{p}}}(\mathbb{1})$ isomorphic to $\left(\mathrm{R}_{\mathscr{K}}\right)_{\mathfrak{p}}$.

\section{Local tensor triangulated categories}

Definition 4.1 A tensor triangulated category $\mathscr{K}$ is local if $\mathrm{Spc}(\mathscr{K})$ is a local topological space, that is, if any open cover $\operatorname{Spc}(\mathscr{K})=\bigcup_{i \in I} U_{i}$ is trivial, in that there exists $i \in I$ such that $U_{i}=\operatorname{Spc}(\mathscr{K})$.

Proposition 4.2 The following are equivalent:

(i) The tensor triangulated category $\mathscr{K}$ is local (Definition 4.1).

(ii) The space $\operatorname{Spc}(\mathscr{K})$ has a unique closed point.

(iii) The category $\mathscr{K}$ has a unique minimal prime.

(iv) The ideal $\sqrt[\otimes]{0} \subset \mathscr{K}$ of $\otimes$-nilpotent objects is prime (and is the minimal one).

(v) For any objects $a, b \in \mathscr{K}$, if $a \otimes b=0$ then $a$ or $b$ is $\otimes$-nilpotent.

If $\mathscr{K}$ is moreover rigid (Definition 1.5), then the above are further equivalent to:

(vi) If $a \otimes b=0$ then $a=0$ or $b=0$. (In this case, 0 is the minimal prime.)

Proof (i) $\Rightarrow$ (ii) If $\mathscr{P}$ and $\mathscr{P}^{\prime}$ were distinct closed points, then the cover $\operatorname{Spc}(\mathscr{K})=$ $(\operatorname{Spc}(\mathscr{K})-\{\mathscr{P}\}) \cup\left(\operatorname{Spc}(\mathscr{K})-\left\{\mathscr{P}^{\prime}\right\}\right)$ would contradict the local nature of $\operatorname{Spc}(\mathscr{K})$.

(ii) $\Rightarrow$ (i) Recall that any nonempty closed subset of $\operatorname{Spc}(\mathscr{K})$ contains a closed point, by [1, Corollary 2.12]. So, if there is only one closed point $M$, any open subset $U \subset \operatorname{Spc}(\mathscr{K})$ which contains $\mathcal{M}$ has an empty closed complement, ie $U=\operatorname{Spc}(\mathscr{K})$. 
(ii) $\Leftrightarrow$ (iii) By [1, Proposition 2.9], the closure of a point $\mathscr{P} \in \mathrm{Spc}(\mathscr{K})$ is described by $\overline{\{\mathscr{P}\}}=\{\mathscr{Q} \in \operatorname{Spc}(\mathscr{K}) \mid \mathscr{Q} \subset \mathscr{P}\}$. Hence closed points are just minimal primes.

(iii) $\Leftrightarrow$ (iv) Immediate from $\bigcap_{\mathscr{P} \in \operatorname{Spc}(\mathscr{K})} \mathscr{P}=\sqrt[\otimes]{0}$; see [1, Corollary 2.4]

(iv) $\Leftrightarrow$ (v) Just reformulation since $a \in \sqrt[\otimes]{0}$ if and only if $a^{\otimes n} \in \sqrt[\otimes]{0}$ for some $n$. Finally, when $\mathscr{K}$ is rigid, $\sqrt[\otimes]{0}=0$; see [2, Corollary 2.5].

Example 4.3 For any prime $\mathscr{P} \in \operatorname{Spc}(\mathscr{K})$ the category $\mathscr{K} / \mathscr{P}$ is local. Indeed, in $\mathscr{K} / \mathscr{P}$, the ideal $0=\mathscr{P} / \mathscr{P}$ is a prime.

The reader's intuition from commutative algebra might be puzzled by (iii) or (vi), in Proposition 4.2, which look more like $\mathcal{K}$ being "integral”. Yet, Definition 4.1 is the conceptual one and the following example should lift any doubt.

Example 4.4 For a commutative ring $A$, the category $\mathrm{K}^{\mathrm{b}}(A-$ proj) is local if and only if $A$ is local. Indeed, $\operatorname{Spc}\left(\mathrm{K}^{\mathrm{b}}(A-\operatorname{proj})\right)$ is homeomorphic to $\operatorname{Spec}(A)$ via

$$
\begin{aligned}
\operatorname{Spec}(A) & \stackrel{\sim}{\longrightarrow} \operatorname{Spc}\left(\mathrm{K}^{\mathrm{b}}(A-\operatorname{proj})\right) \\
\mathfrak{p} & \longmapsto \mathscr{P}(\mathfrak{p}):=\left\{M_{\bullet} \in \mathrm{K}^{\mathrm{b}}(A-\operatorname{proj}) \mid\left(M_{\bullet}\right)_{\mathfrak{p}} \simeq 0\right\}
\end{aligned}
$$

by [1, Corollary 6.3 (a)] and [9, Theorem 8.5$]$. Now, $\operatorname{Spec}(A)$ has a unique closed point if and only if $A$ is local. The subtlety is that the above map is inclusion-reversing: $\mathfrak{p} \subset \mathfrak{q}$ implies $\mathscr{P}(\mathfrak{p}) \supset \mathscr{P}(\mathfrak{q})$.

Theorem 4.5 If the tensor triangulated category $\mathscr{K}$ is local (Definition 4.1), then $\mathrm{R}_{\mathscr{K}}^{\bullet}=\operatorname{End}_{\mathscr{K}, u}^{\bullet}(\mathbb{1})$ is a local graded ring. In particular, $\mathrm{R}_{\mathscr{K}}=\operatorname{End}_{\mathscr{K}_{\ell}}(\mathbb{1})$ is a local ring.

Proof We have to prove that $\mathrm{R}_{\mathscr{K}}^{\bullet}$ admits a unique maximal homogeneous ideal, which must then be the ideal generated by the noninvertible homogeneous elements. Since the product of a noninvertible with any other element remains noninvertible, it suffices to check that the sum of two noninvertible elements of same degree remains noninvertible. Let $f, g \in \mathrm{R}_{\mathscr{K}}^{d}$, for some $d \in \mathbb{Z}$, be such that $f+g$ is invertible and let us prove that $f$ or $g$ is. In Notation 2.6, consider the morphism

$$
(f+g) l_{\text {cone }(f) \otimes \operatorname{cone}(g)}: \operatorname{cone}(f) \otimes \operatorname{cone}(g) \longrightarrow u^{\otimes d} \otimes \operatorname{cone}(f) \otimes \operatorname{cone}(g) .
$$

We claim that this morphism is both invertible and nilpotent in the graded ring $\operatorname{End}_{\mathscr{K}}^{\bullet}(\operatorname{cone}(f) \otimes \operatorname{cone}(g))$. The former is clear since $f+g$ is invertible by assumption. To prove nilpotence, since $f$ and $g \epsilon_{u}$-commute, it suffices to show that both $f$ and $g$ are nilpotent on cone $(f) \otimes \operatorname{cone}(g)$. By Proposition 3.3, $\left(f l_{\operatorname{cone}(f) \otimes \operatorname{cone}(g)}\right)^{2}=$ $f \otimes f \otimes \mathrm{id}_{\text {cone }(f)} \otimes \mathrm{id}_{\text {cone }(g)}=0$ by Proposition 2.13, and similarly for $g$. Hence the 
morphism $(f+g)^{3} L_{\text {cone }}(f) \otimes \operatorname{cone}(g)$ is both invertible and zero. This forces cone $(f) \otimes$ cone $(g)=0$. But $\mathscr{K}$ is local, so by Proposition $4.2(\mathrm{v})$, we have cone $(f)^{\otimes n}=0$, or cone $(g)^{\otimes n}=0$, for $n \in \mathbb{N}$ big enough. By Proposition 2.16, we deduce cone $(f)=0$ or cone $(g)=0$, ie $f$ or $g$ is an isomorphism.

The second statement follows: For any $R^{\bullet}$ local graded ring, $R^{0}$ is local.

Example 4.6 The converse to Theorem 4.5 does not hold. Consider $\mathscr{K}=\mathrm{D}^{\text {perf }}(X)$ for a nonlocal scheme $X$. It can nevertheless happen that $\operatorname{End}_{\mathscr{K}}(\mathbb{1})=\Gamma\left(X, \mathcal{O}_{X}\right)$ is local, say, when $\mathcal{O}_{X}$ does not have many global sections, eg for $X=\mathbb{P}_{k}^{n}$.

\section{From triangular spectrum to Zariski spectra}

Let $\mathscr{K}$ be a tensor triangulated category. As before, we tacitly fix an invertible object $u \in \mathscr{K}$, like $u=\Sigma(\mathbb{1})$, for instance. Recall the graded central ring $\mathrm{R}_{\mathscr{K}}^{\bullet}=$ End $_{\mathscr{K}}^{\bullet}(\mathbb{1})=$ $\operatorname{Hom}_{\mathscr{K}}\left(\mathbb{1}, u^{\otimes \bullet}\right)$ of Section 3 .

Definition 5.1 Let $\mathscr{P} \in \operatorname{Spc}(\mathscr{K})$ be a triangular prime. We define $\rho_{\mathscr{K}}^{\bullet}(\mathscr{P}) \subset \mathrm{R}_{\mathscr{K}}^{\bullet}$ as the following homogeneous ideal (given by homogeneous generators):

$$
\rho_{\mathscr{K}}^{\bullet}(\mathscr{P}):=\left\langle f \in \mathrm{R}_{\mathscr{K}}^{\text {hom }} \mid \operatorname{cone}(f) \notin \mathscr{P}\right\rangle .
$$

Remark 5.2 Observe that $\rho_{\mathscr{K}}^{\bullet}$ "reverses" inclusions: If $\mathscr{P} \subset \mathcal{Q}$ are two primes of $\mathscr{K}$, then $\rho_{\mathscr{K}}^{\bullet}(\mathscr{P}) \supset \rho_{\mathscr{K}}^{\bullet}(2)$ in $\mathrm{R}_{\mathscr{K}}^{\bullet}$.

Theorem 5.3 Let $\mathscr{K}$ be a tensor triangulated category. Let $u \in \mathscr{K}$ be an invertible object and recall the graded central ring $\mathrm{R}_{\mathscr{K}}^{\bullet}=\mathrm{R}_{\mathscr{K}, u}^{\bullet}$ of Definition 3.2.

(a) Let $\mathscr{P} \in \operatorname{Spc}(\mathscr{K})$. The subset $\rho_{\mathscr{K}}^{\bullet}(\mathscr{P}) \subset \mathrm{R}_{\mathscr{K}}^{\bullet}$ is a homogeneous prime ideal, equal to the preimage under the localization homomorphism $\mathrm{R}_{\mathscr{K}}^{\bullet} \rightarrow \mathrm{R}_{\mathscr{K} / \mathscr{P}}^{\bullet}$ of the maximal homogeneous ideal of $\mathrm{R}_{\mathscr{K} / \mathscr{P}}^{\bullet}$.

(b) The map $\rho_{\mathscr{K}}^{\bullet}: \operatorname{Spc}(\mathscr{K}) \rightarrow \operatorname{Spec}^{\mathrm{h}}\left(\mathrm{R}_{\mathscr{K}}^{\bullet}\right)$ is continuous. More precisely, for any $s \in \mathrm{R}_{\mathscr{K}}^{\bullet}$ homogeneous, the preimage of the principal open $D(s) \subset \operatorname{Spec}^{\mathrm{h}}\left(\mathrm{R}_{\mathscr{K}}^{\bullet}\right)$ is the open $U(\operatorname{cone}(s)) \subset \operatorname{Spc}(\mathscr{K})$; see Definition 1.3.

(c) The above defines a natural transformation $\rho^{\bullet}$ between the contravariant functors $\mathscr{K} \mapsto \operatorname{Spc}(\mathscr{K})$ and $\mathscr{K} \mapsto \operatorname{Spec}^{\mathrm{h}}\left(\mathrm{R}_{\mathscr{K}}^{\bullet}\right)$ from the category of tensor triangulated categories (with tensor triangulated functors respecting the chosen invertible objects) to the category of topological spaces. 
Proof Consider the localization functor $q: \mathscr{K} \longrightarrow \mathscr{L}:=\mathscr{K} / \mathscr{P}$. Choose $q(u)$ as invertible in $\mathscr{L}$. The functor $q$ gives us in particular a ring homomorphism $f \mapsto q(f)$

$$
\mathrm{R}_{\mathscr{K}}^{\bullet}=\operatorname{Hom}_{\mathscr{K}}^{\bullet}\left(\mathbb{1}_{\mathscr{K}}, \mathbb{1}_{\mathscr{K}}\right) \stackrel{q}{\longrightarrow} \operatorname{Hom}_{\mathscr{L}}^{\bullet}\left(\mathbb{1}_{\mathscr{L}}, \mathbb{1}_{\mathscr{L}}\right)=\mathrm{R}_{\mathscr{L}}^{\bullet} .
$$

Since the category $\mathscr{L}$ is local (Example 4.3 ) we know by Theorem 4.5 that $\mathrm{R}_{\mathscr{L}}^{\bullet}$ is a local graded ring, with maximal ideal $\mathfrak{m}^{\bullet}:=\left\langle f \in \mathrm{R}_{\mathscr{L}}^{\text {hom }}\right| f$ is not invertible $\rangle$. For a morphism $f$ in $\mathscr{K}$, since $q: \mathscr{K} \longrightarrow \mathscr{L}$ is exact, we have cone $(q(f)) \simeq q($ cone $(f))$ and for an object $x \in \mathscr{K}$, we have $q(x)=0$ if and only if $x \in \mathscr{P}$. Therefore $\rho_{\mathscr{K}}^{\bullet}(\mathscr{P})=$ $\left\langle f \in \mathrm{R}_{\mathscr{K}}^{\text {hom }}\right| q(f)$ is not invertible $\rangle=q^{-1}\left(\mathfrak{m}^{\bullet}\right)$. So, $\rho_{\mathscr{K}}^{\bullet}(\mathscr{P})$ is the image of $\mathfrak{m}^{\bullet}$ by the $\operatorname{map} \operatorname{Spec}^{\mathrm{h}}(q): \operatorname{Spec}^{\mathrm{h}}\left(\mathrm{R}_{\mathscr{L}}^{\bullet}\right) \rightarrow \operatorname{Spec}^{\mathrm{h}}\left(\mathrm{R}_{\mathscr{K}}^{\bullet}\right)$. Hence (a).

For (b), unfolding the definitions, we see that $\mathscr{P} \in\left(\rho_{\mathscr{K}}^{\bullet}\right)^{-1}(D(s))$ if and only if cone $(s) \in$ $\mathscr{P}$, that is, $\mathscr{P} \in U($ cone $(s))$ see Definition 1.3.

Finally, for (c), let $F: \mathscr{K} \rightarrow \mathscr{K}^{\prime}$ be a morphism of tensor triangulated categories, that is, an exact $\otimes$-functor. We have the fixed invertible objects $u$ in $\mathscr{K}$ and $u^{\prime}=F(u)$ in $\mathscr{K}^{\prime}$. Let us also denote by $F: \mathrm{R}_{\mathscr{K}}^{\bullet} \rightarrow \mathrm{R}_{\mathscr{K}^{\prime}}^{\bullet}, f \mapsto F(f)$, the induced ring homomorphism. We need to prove the commutativity of the following diagram:

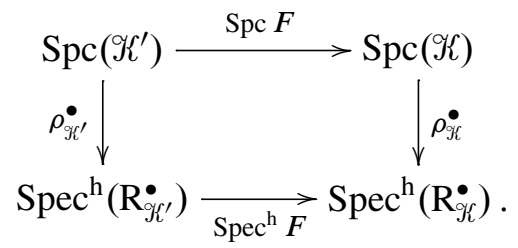

Let $\mathscr{P}^{\prime} \in \operatorname{Spc}\left(\mathscr{K}^{\prime}\right)$ and recall that $(\operatorname{Spc} F)\left(\mathscr{P}^{\prime}\right)=F^{-1}\left(\mathscr{P}^{\prime}\right)$. For $f \in \mathrm{R}_{\mathscr{K}}^{\text {hom }}$, unfolding the definitions, we have $f \in \rho_{\mathscr{K}}^{\bullet}\left((\operatorname{Spc} F)\left(\mathscr{P}^{\prime}\right)\right) \Leftrightarrow \operatorname{cone}(f) \notin(\operatorname{Spc} F)\left(\mathscr{P}^{\prime}\right)=F^{-1}\left(\mathscr{P}^{\prime}\right) \Leftrightarrow$ $F$ (cone $(f)) \notin \mathscr{P}^{\prime}$. Now, $F$ being exact, $F(\operatorname{cone}(f))=\operatorname{cone}(F(f))$ and the condition becomes equivalent to cone $(F(f)) \notin \mathscr{P}^{\prime} \Leftrightarrow F(f) \in \rho_{\mathscr{K}^{\prime}}^{\bullet}\left(\mathscr{P}^{\prime}\right) \Leftrightarrow f \in F^{-1}\left(\rho_{\mathscr{K}^{\prime}}^{\bullet}\left(\mathscr{P}^{\prime}\right)\right)=$ $\left(\operatorname{Spec}^{\mathrm{h}} F\right)\left(\rho_{\mathscr{K}^{\prime}}^{\bullet}\left(\mathscr{P}^{\prime}\right)\right)$.

Theorem 5.4 Let $S \subset \mathrm{R}_{\mathscr{K}}^{\bullet}$ be a central homogeneous multiplicative subset and let $q: \mathscr{K} \rightarrow S^{-1} \mathscr{K}$ be the corresponding localization (see Theorem 3.6). Then the diagram

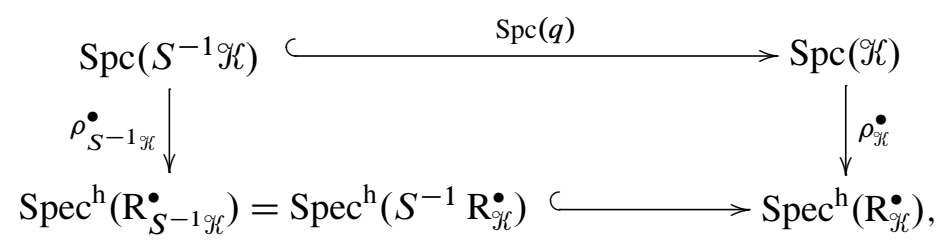

commutes and is cartesian: $\operatorname{Spc}\left(S^{-1 \mathscr{K}}\right) \cong\left\{\mathscr{P} \in \operatorname{Spc}(\mathscr{K}) \mid S \cap \rho_{\mathscr{K}}^{\bullet}(\mathscr{P})=\varnothing\right\}$. 
Proof The diagram commutes by naturality of $\rho^{\bullet}$ (Theorem 5.3 (c)). As in Theorem 3.6, let $\mathscr{g}=\langle\operatorname{cone}(s) \mid s \in S\rangle$, so that $S^{-1 \mathscr{K}}=\mathscr{K} / \mathscr{F}$. We know that $\operatorname{Spc}\left(S^{-1 \mathscr{K}}\right)$ is homeomorphic, via $\operatorname{Spc}(q)$, to $\{\mathscr{P} \in \operatorname{Spc}(\mathscr{K}) \mid \mathscr{\mathscr { C }} \subset \mathscr{P}\}$; see Remark 1.4. By definition of $\mathscr{E}$, we have $\mathscr{S} \subset \mathscr{P}$ if and only if cone $(s) \in \mathscr{P}$ for all $s \in S$, which is equivalent to $s \notin \rho_{\mathscr{K}}^{\bullet}(\mathscr{P})$ for all $s \in S$, by Definition 5.1.

Remark 5.5 For each $\mathbb{Z}$-graded $\epsilon$-commutative ring $R^{\bullet}$, we have a continuous map $(-)^{0}: \operatorname{Spec}^{\mathrm{h}}\left(R^{\bullet}\right) \longrightarrow \operatorname{Spec}\left(R^{0}\right), \mathfrak{p}^{\bullet} \mapsto \mathfrak{p}^{0}=\mathfrak{p}^{\bullet} \cap R^{0}$. This is natural in $R^{\bullet}$, with respect to homogeneous ring homomorphisms. Note that this map is surjective. Indeed, let $\mathfrak{p} \in \operatorname{Spec}\left(R^{0}\right)$. We can localize at $\mathfrak{p}$ (invert $S=R^{0} \backslash \mathfrak{p}$ ) and reduce to the case where $R^{0}$ is local with maximal ideal $\mathfrak{p}$. Then any homogeneous prime $\mathfrak{q}^{\bullet}$ containing the proper ideal $\mathfrak{p} \cdot R^{\bullet}$ (eg a maximal one) will satisfy $\mathfrak{q}^{\bullet} \cap R^{0}=\mathfrak{p}$ : One inclusion since $\mathfrak{q}^{\bullet}$ contains $\mathfrak{p}$ and the other one since $\mathfrak{p}$ is maximal. Composing $\rho_{\mathscr{K}}^{\bullet}$ with the map $(-)^{0}$ yields the following corollary.

Corollary 5.6 Let $\mathscr{K}$ be a tensor triangulated category and let $\mathscr{P} \in \operatorname{Spc}(\mathscr{K})$. We define $\rho_{\mathscr{K}}(\mathscr{P}) \subset \mathrm{R}_{\mathscr{K}}=\operatorname{End}_{\mathscr{K}}(\mathbb{1})$ as the subset

$$
\rho_{\mathscr{K}}(\mathscr{P}):=\left\{f \in \mathrm{R}_{\mathscr{K}} \mid \operatorname{cone}(f) \notin \mathscr{P}\right\} .
$$

(This does not rely on the choice of an invertible object $u \in \mathscr{K}$.) Then we have:

(a) For each $\mathscr{P} \in \operatorname{Spc}(\mathscr{K})$, the subset $\rho_{\mathscr{K}}(\mathscr{P}) \subset \mathrm{R}_{\mathscr{K}}$ is a prime ideal.

(b) The map $\rho_{\mathscr{K}}: \operatorname{Spc}(\mathscr{K}) \rightarrow \operatorname{Spec}\left(\mathrm{R}_{\mathscr{K}}\right)$ is continuous, natural in $\mathscr{K}$, and the following diagram commutes:

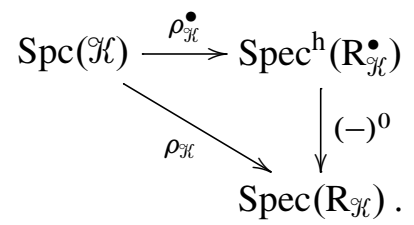

(c) Let $S \subset \mathrm{R}_{\mathscr{K}}$ be a multiplicative subset and let $q$ : $\mathscr{K} \rightarrow S^{-1} \mathscr{K}$ be the corresponding localization (see Corollary 3.10). Then the commutative diagram

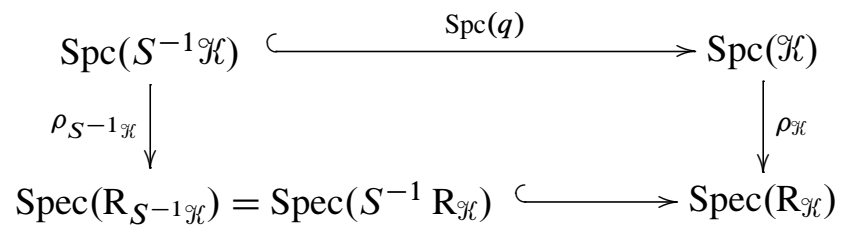

is cartesian, ie $\operatorname{Spc}\left(S^{-1} \mathscr{K}\right) \cong\left\{\mathscr{P} \in \operatorname{Spc}(\mathscr{K}) \mid S \cap \rho_{\mathscr{K}}(\mathscr{P})=\varnothing\right\}$. 
Proof The diagram in (b) commutes by definition; see (5-1). Therefore (a) and the rest of (b) follow. Only (c) requires some comment. Indeed, it follows from Theorem 5.4 and the following facts: For any graded ring $R^{\bullet}$ and any multiplicative subset $S \subset R^{0}$, we have $\left(S^{-1} R^{\bullet}\right)^{0}=S^{-1} R^{0}$ and the commutative diagram

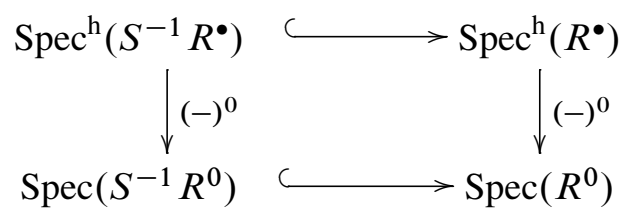

is cartesian. Indeed, for $\mathfrak{p}^{\bullet} \in \operatorname{Spec}^{\mathrm{h}}\left(R^{\bullet}\right)$ we have $\mathfrak{p}^{\bullet} \cap S=\varnothing \Leftrightarrow \mathfrak{p}^{0} \cap S=\varnothing$.

Remark 5.7 Not only are the spaces $\operatorname{Spc}(\mathscr{K}), \operatorname{Spec}^{\mathrm{h}}\left(\mathrm{R}_{\mathscr{K}}^{\bullet}\right)$ and $\operatorname{Spec}\left(\mathrm{R}_{\mathscr{K}}\right)$ spectral in the sense of Hochster [14] but Theorem 5.3 (b) shows that $\rho_{\mathscr{K}}^{\bullet}$ and $\rho_{\mathscr{Y}}$ are spectral maps, namely the preimage of a quasicompact open is quasicompact.

\section{Locally ringed space structures}

The spectrum $\operatorname{Spc}(\mathscr{K})$ of a tensor triangulated category $\mathscr{K}$ can be turned into a ringed space, as explained in [1, Section 6]. We recall the definition, introduce a graded version of it, and then compare these structure sheaves with the ones on $\operatorname{Spec}\left(\mathrm{R}_{\mathscr{K}}\right)$ and on $\operatorname{Spec}^{\mathrm{h}}\left(\mathrm{R}_{\mathscr{K}}^{\bullet}\right)$; see Remark 3.4. More important than the sheaf of rings, the fundamental geometric feature is the following "presheaf" of triangulated categories.

Throughout this section, $\mathscr{K}$ is a rigid (Definition 1.5) tensor triangulated category. We use this assumption to deduce that any thick triangulated $\otimes$-ideal $\mathscr{\mathscr { S }}$ of $\mathscr{K}$ is radical, that is $x^{\otimes n} \in \mathscr{F}$ implies $x \in \mathscr{F}$ (see [1, Proposition 2.4]).

Construction 6.1 Let $U \subset \operatorname{Spc}(\mathscr{K})$ be a quasicompact open with closed complement $Z$. Define $\mathscr{K}_{Z}:=\{a \in \mathscr{K} \mid \operatorname{supp}(a) \subset Z\}$ to be the thick triangulated $\otimes$-ideal of $\mathscr{K}$ supported outside $U$. Then define the tensor triangulated category " $\mathscr{K}$ on $U$ "

$$
\mathscr{K}(U):=\left(\mathscr{K}_{/} / \mathscr{K}_{Z}\right)^{\natural}
$$

as the idempotent completion $(-)^{\natural}$ of the Verdier quotient $\mathscr{K}_{K} / \mathscr{K}_{Z}$. This quotient is the localization $S^{-1} \mathscr{K}$ with respect to $S=\{s: a \rightarrow b \mid \operatorname{supp}(\operatorname{cone}(s)) \subset Z\}$. By construction we have a natural functor

$$
q_{U}: \mathscr{K} \longrightarrow \mathscr{K}(U) .
$$

It sends in particular the unit $\mathbb{1}=\mathbb{1}_{\mathscr{K}} \in \mathscr{K}$ to the unit $\mathbb{1}_{\mathscr{K}(U)}$, which we simply denote $\mathbb{1}_{U}$. We will consider endomorphism rings of these unit objects. The above idempotent 
completion is harmless for the sequel since the inclusion $\mathscr{K} \hookrightarrow \mathscr{K}^{\natural}$ is fully faithful but (6-1) is the right definition of $\mathscr{K}(U)$ in view of geometric examples; see more in [1, Remark 6.2] including the reference to Thomason's localization theorem.

Remark 6.2 The space $\operatorname{Spc}(\mathscr{K})$ has a basis of quasicompact open subsets; see [1, Remark 2.7 and Proposition 2.14]. We tacitly use this fact everywhere by describing our presheaves on such opens only. This is enough for the associated sheaves anyway. We focus on quasicompact opens when defining $\mathscr{K}(U)$ because $\operatorname{Spc}(\mathscr{K}(U)) \cong U$ if and only if $U$ is quasicompact; see [1, Corollary 2.15] and [6, Proposition 1.11].

Lemma 6.3 Let $\mathscr{P} \in \operatorname{Spc}(\mathscr{K})$ and let $a, b \in \mathscr{K}$. There is a natural isomorphism $\operatorname{colim}_{U \ni \mathscr{P}} \operatorname{Hom}_{\mathscr{K}(U)}(a, b) \stackrel{\sim}{\rightarrow} \operatorname{Hom}_{\mathscr{K} / \mathscr{P}}(a, b)$, where the colimit is taken over the quasicompact open subsets of $\operatorname{Spc}(\mathscr{K})$ containing the point $\mathscr{P}$.

Proof Since we are considering $a, b \in \mathscr{K}$, and since idempotent completion is fully faithful, we have for every $U$ that $\operatorname{Hom}_{\mathscr{K}(U)}(a, b)=\operatorname{Hom}_{\mathscr{K}_{/} / \mathscr{K}_{Z}}(a, b)$ where $Z=$ $\operatorname{Spc}(\mathscr{K}) \backslash U$. A morphism in the localization $\mathscr{K} / \mathscr{K}_{Z}$ is the equivalence class of a fraction $a \stackrel{s}{\leftarrow} x \rightarrow b$ where $\operatorname{supp}(\operatorname{cone}(s)) \subset Z$, the equivalence being with respect to amplification, as usual. Letting $U$ shrink among the quasicompact opens containing $\mathscr{P}$, it is easy to check that the stated colimit amounts to the equivalence classes of fractions $a \stackrel{s}{\leftarrow} x \rightarrow b$ where $\operatorname{supp}(\operatorname{cone}(s)) \subset \operatorname{Spc}(\mathscr{K}) \backslash\{\mathscr{P}\}$, which is equivalent to cone $(s) \in \mathscr{P}$. Such fractions precisely describe the morphisms in $\mathscr{K} / \mathscr{P}$.

Definition 6.4 For each quasicompact open $U \subset \operatorname{Spc}(\mathscr{K})$, define the commutative ring $\mathrm{p} \mathcal{O}_{\mathscr{K}}(U)$ and the $\epsilon_{u}$-commutative $\mathbb{Z}$-graded ring $\mathrm{p}_{\mathscr{F}_{\mathcal{K}}}^{\bullet}(U)$ by

$$
\mathrm{p}_{\mathcal{K}}(U):=\mathrm{R}_{\mathscr{K}(U)}=\operatorname{End}_{\mathscr{K}(U)}\left(\mathbb{1}_{U}\right) \quad \text { and } \quad \mathrm{p}_{\mathscr{\mathscr { K }}}^{\bullet}(U):=\mathrm{R}_{\mathscr{K}(U)}^{\bullet} .
$$

For the right-hand graded ring (Definition 3.2), some invertible object $u \in \mathscr{K}$ is assumed fixed beforehand, for instance $u=\Sigma(\mathbb{1})$ and we use its image in $\mathscr{K}(U)$. These $\mathrm{p} \mathcal{O}_{\mathscr{K}}$ and $\mathrm{O}_{\mathscr{K}}^{\bullet}$ are partially-defined presheaves on $\operatorname{Spc}(\mathscr{K})$, only defined on an open basis; see Remark 6.2. Their associated sheaves on the space $\operatorname{Spc}(\mathscr{K})$ are denoted

$$
\mathcal{O}_{\mathscr{K}}:=\widetilde{\mathrm{p} \mathcal{O}_{\mathscr{K}}} \quad \text { and } \quad \mathcal{O}_{\mathscr{K}}^{\bullet}:=\widetilde{\mathrm{p} \mathcal{O}_{\mathscr{K}}^{\bullet}} \text {. }
$$

Finally, we denote the ringed spaces thus obtained by

$$
\operatorname{Spec}(\mathscr{K}):=\left(\operatorname{Spc}(\mathscr{K}), \mathcal{O}_{\mathscr{K}}\right) \text { and } \operatorname{Spec}^{\bullet}(\mathscr{K}):=\left(\operatorname{Spc}(\mathscr{K}), \mathcal{O}_{\mathscr{K}}^{\bullet}\right) \text {. }
$$

Note that they are both defined on the same space, namely the spectrum of $\mathscr{K}$. 
Proposition 6.5 Let $\mathscr{P} \in \operatorname{Spc}(\mathscr{K})$. The stalk of $\mathcal{O}_{\mathscr{K}}^{\bullet}$ and of $\mathrm{p} \mathcal{O}_{\mathscr{K}}^{\bullet}$ at $\mathscr{P}$ are naturally isomorphic to $\mathrm{R}_{\mathscr{K} / \mathscr{P}}^{\bullet}$. In particular, the stalk of $\mathcal{O}_{\mathscr{K}}$ and of $\mathrm{p} \mathcal{O}_{\mathscr{K}}$ at $\mathscr{P}$ are naturally isomorphic to $\mathrm{R}_{\mathscr{K} / \mathscr{P}}$.

Proof It follows directly from Lemma 6.3 applied to $a=\mathbb{1}$ and $b=u^{\otimes i}$ that the stalk at $\mathscr{P}$ of the presheaf $U \mapsto \mathrm{R}_{\mathscr{K}(U)}^{i}$ is $\mathrm{R}_{\mathscr{K} / \mathscr{P}}^{i}$ for all $i \in \mathbb{Z}$. This gives the result for the presheaves $\mathrm{p} \mathcal{O}_{\mathscr{K}}$ and $\mathrm{p} \mathcal{O}_{\mathscr{K}}^{\bullet}$, hence for the associated sheaves $\mathcal{O}_{\mathscr{K}}$ and $\mathcal{O}_{\mathscr{K}}^{\bullet}$.

Corollary 6.6 The ringed space $\operatorname{Spec}(\mathscr{K})$ is a locally ringed space.

Proof By Proposition 6.5, it suffices to prove that $\mathrm{R}_{\mathscr{K} / \mathscr{P}}$ is a local ring. This holds by Theorem 4.5 since the category $\mathscr{K} / \mathscr{P}$ is local (see Example 4.3).

Remark 6.7 The author doesn't know whether the concept of "locally graded ringed space" exists but the proof of Corollary 6.6 applies to $\operatorname{Spec}^{\bullet}(\mathscr{K})$ as well, showing that the stalks of $\mathcal{O}_{\mathscr{K}}^{\bullet}$ are local in the graded sense.

Remark 6.8 We now want to show that the continuous maps $\rho_{\mathscr{K}}: \operatorname{Spc}(\mathscr{K}) \rightarrow \operatorname{Spec}\left(\mathrm{R}_{\mathscr{K}}\right)$ and $\rho_{\mathscr{K}}^{\bullet}: \operatorname{Spc}(\mathscr{K}) \rightarrow \operatorname{Spec}^{\mathrm{h}}\left(\mathrm{R}_{\mathscr{K}}^{\bullet}\right)$ of Section 5 can be upgraded into morphisms of locally ringed spaces.

Recall that a morphism $\rho:\left(X, \mathcal{O}_{X}\right) \rightarrow\left(Y, \mathcal{O}_{Y}\right)$ of ringed spaces consists of a continuous map $\rho: X \rightarrow Y$ together with ring homomorphisms $r_{U}: \mathcal{O}_{Y}(U) \rightarrow \mathcal{O}_{X}\left(\rho^{-1}(U)\right)$ for all open $U \subset Y$, in a compatible way with the restrictions. As usual, it is enough to do so on a basis of the topology of the base space $Y$.

Lemma 6.9 For every homogenous $s \in \mathrm{R}_{\mathscr{K}}^{\text {even }}$ there is a natural isomorphism between the sections of the sheaf $\mathcal{O}_{\operatorname{Spec}^{\mathrm{h}}\left(\mathrm{R}_{\mathscr{K}}^{\bullet}\right)}$ over the principal open $D(s)$ of $\operatorname{Spec}^{\mathrm{h}}\left(\mathrm{R}_{\mathscr{K}}^{\bullet}\right)$ and the sections of the presheaf $\mathrm{p} \mathcal{O}_{\mathscr{K}}^{\bullet}$ over the preimage $\left(\rho_{\mathscr{K}}^{\bullet}\right)^{-1}(D(s))=U(\operatorname{cone}(s))$. They are both naturally isomorphic to $\mathrm{R}_{\mathscr{K}}^{\bullet}[1 / s]$.

There is an analogous obvious ungraded statement for $s \in \mathrm{R}_{\mathscr{K}}$ mutatis mutandis.

Proof By definition, $\mathcal{O}_{\dot{S p e c}^{\mathrm{h}}\left(\mathrm{R}_{\mathscr{f}}^{\bullet}\right)}(D(s))=\mathrm{R}_{\mathscr{K}}^{\bullet}[1 / s]$. On the other hand, we know by Theorem 5.3 (b) that the preimage of $D(s)$ under $\rho_{\mathscr{K}}^{\bullet}: \operatorname{Spc}(\mathscr{K}) \rightarrow \operatorname{Spec}^{\mathrm{h}}\left(\mathrm{R}_{\mathscr{K}}^{\bullet}\right)$ is $U(s):=U(\operatorname{cone}(s))$. Finally $\mathrm{p}_{\mathscr{\mathscr { K }}}^{\bullet}(U(s))=\mathrm{R}_{\mathscr{K}(U(s))}$ can be computed by Theorem

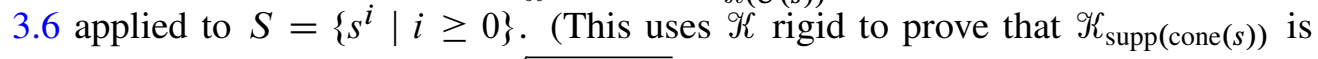
indeed $\langle\operatorname{cone}(s)\rangle$ and not just $\sqrt[\otimes]{\langle\operatorname{cone}(s)\rangle}$.) This yields $\mathrm{R}_{\mathscr{K}}^{\bullet}[1 / s]$ as well. Hence the result. The ungraded statement follows by contemplating degree zero only. 
Construction 6.10 For every $s \in \mathrm{R}_{\mathscr{K}}^{\text {even }}$, let $U(s):=U(\operatorname{cone}(s))=\left(\rho_{\mathscr{K}}^{\bullet}\right)^{-1}(D(s)) \subset$ $\operatorname{Spc}(\mathscr{K})$ and define the ring homomorphism

$$
r_{D(s)}: \mathcal{O}_{\operatorname{Spec}^{\mathrm{h}}\left(\mathrm{R}_{\mathscr{f}}^{\bullet}\right)}(D(s)) \longrightarrow \mathcal{O}_{\mathscr{K}}^{\bullet}(U(s))
$$

as the composite of the isomorphism $\mathcal{O}_{\operatorname{Spec}^{\mathrm{h}}\left(\mathrm{R}_{\mathscr{C}}^{\bullet}\right)}^{\bullet}(D(s)) \stackrel{\sim}{\rightarrow} \mathrm{p}_{\mathscr{K}}^{\bullet}(U(s))$ of Lemma 6.9 followed by the (sheafification) morphism $\mathrm{p}_{\mathscr{K}}^{\circ} \rightarrow \mathcal{O}_{\mathscr{K}}^{\bullet}$ on $U(s)$. This construction is compatible with restriction and defines a morphism of ringed spaces

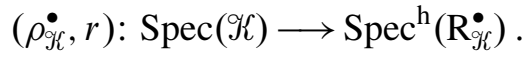

Concentrating on the degree zero part (hence allowing $s \in \mathrm{R}_{\mathscr{C}}$ only), we obtain a morphism of ringed spaces $\left(\rho_{\mathscr{K}}, r\right): \operatorname{Spc}(\mathscr{K}) \rightarrow \operatorname{Spec}\left(\mathrm{R}_{\mathscr{K}}\right)$.

Proposition 6.11 The above morphisms are morphisms of locally ringed spaces. Moreover, we have the following properties:

(a) For a prime $\mathscr{P} \in \operatorname{Spc}(\mathscr{K})$, let $\mathfrak{p}=\rho_{\mathscr{K}}(\mathscr{P}) \in \operatorname{Spec}\left(\mathrm{R}_{\mathscr{K}}\right)$ and $\mathfrak{p}^{\bullet}=\rho_{\mathscr{K}}^{\bullet}(\mathscr{P}) \in$ $\operatorname{Spec}^{\mathrm{h}}\left(\mathrm{R}_{\mathscr{K}}^{\bullet}\right)$. Then the induced homomorphisms on stalks are the natural ones, $\left(\mathrm{R}_{\mathscr{K}}\right)_{\mathfrak{p}} \rightarrow \mathrm{R}_{\mathscr{K} / \mathscr{P}}$ and $\left(\mathrm{R}_{\mathscr{K}}^{\bullet}\right)_{\mathfrak{p}} \bullet \rightarrow \mathrm{R}_{\mathscr{K} / \mathscr{P}}^{\bullet}$, induced by localization at $\mathscr{P}$.

(b) If $\rho_{\mathscr{K}}$ or $\rho_{\mathscr{K}}^{\bullet}$ is a homeomorphism on the underlying spaces, it is automatically an isomorphism of ringed spaces.

Proof We prove the graded version. The ungraded version can be proved similarly. For readability, let us abbreviate $\rho:=\rho_{\mathscr{K}}^{\bullet}: \operatorname{Spc}(\mathscr{K}) \rightarrow \operatorname{Spec}^{\mathrm{h}}\left(\mathrm{R}_{\mathscr{K}}^{\bullet}\right)$ on spaces.

Localization $q_{\mathscr{P}}: \mathscr{K} \rightarrow \mathscr{K} / \mathscr{P}$ induces a ring homomorphism $\mathrm{R}_{\mathscr{K}}^{\bullet} \rightarrow \mathrm{R}_{\mathscr{K} / \mathscr{P}}$. By Theorem 5.3 (a), the preimage under this homomorphism of the maximal ideal of $\mathrm{R}_{\mathscr{K} / \mathscr{P}}^{\bullet}$ is precisely the ideal $\mathfrak{p}^{\bullet}=\rho(\mathscr{P}) \subset \mathrm{R}_{\mathscr{K}}^{\bullet}$. Hence, the induced morphism of local rings $\ell:\left(\mathrm{R}_{\mathscr{K}}^{\bullet}\right)_{\mathfrak{p}} \bullet \rightarrow \mathrm{R}_{\mathscr{K} / \mathscr{P}}^{\bullet}$ is local (the image of the maximal ideal of the source is contained in the maximal ideal of the target). We claim that this morphism $\ell:\left(\mathrm{R}_{\mathscr{K}}^{\bullet}\right)_{\mathfrak{p}} \bullet \rightarrow \mathrm{R}_{\mathscr{K} / \mathscr{P}}^{\bullet}$ is precisely the one induced stalkwise by the morphism of ringed spaces $\operatorname{Spec}^{\bullet}(\mathscr{K}) \rightarrow$ $\operatorname{Spec}^{\mathrm{h}}\left(\mathrm{R}_{\mathscr{\mathscr { K }}}^{\bullet}\right)$ above. Recall Proposition 6.5. An element $f / s \in\left(\mathrm{R}_{\mathscr{K}}^{\bullet}\right)_{\mathfrak{p}} \bullet$ defines an element of $\operatorname{End}_{\mathscr{K}(U(s))}^{\bullet}\left(\mathbb{1}_{U(s)}\right)$ whose class in $\mathscr{K} / \mathscr{P}$ is precisely $\ell(f / s)$. This yields Part (a). Hence our morphism of ringed spaces is stalkwise local, so it is a morphism of locally ringed spaces.

Part (b) is then easy to deduce from Lemma 6.9. Indeed, assuming the two spaces are the same, the presheaf $\mathcal{O}_{\mathscr{K}}^{\bullet}$ agrees with the sheaf $\mathcal{O}_{\mathrm{Spec}^{\mathrm{h}}\left(\mathrm{R}_{\mathscr{K}}^{\bullet}\right)}$ on principal opens. Consequently its sheafification $\mathcal{O}_{\mathscr{K}}^{\bullet}$ is exactly $\mathcal{O}_{\operatorname{Spec}^{\mathrm{h}}\left(\mathrm{R}_{\mathscr{K}}^{\bullet}\right)}^{\bullet}$ as claimed.

So much for structure sheaves. We now return to spectra. 


\section{Two criteria for surjectivity}

We want to give conditions for the maps $\rho_{\mathscr{K}}^{\bullet}: \operatorname{Spc}(\mathscr{K}) \rightarrow \operatorname{Spec}^{\mathrm{h}}\left(\mathrm{R}_{\mathscr{K}}^{\bullet}\right)$ and $\rho_{\mathscr{K}}: \operatorname{Spc}(\mathscr{K}) \rightarrow$ $\operatorname{Spec}\left(\mathrm{R}_{\mathscr{K}}\right)$ to be surjective. First, we reduce to the case of $\mathrm{R}_{\mathscr{K}}^{\bullet}$ local.

Proposition 7.1 Let $\mathfrak{p}^{\bullet} \in \operatorname{Spec}^{\mathrm{h}}\left(\mathrm{R}_{\mathscr{K}}^{\bullet}\right)$ be a homogeneous prime. Consider the localization $\mathscr{L}:=\mathscr{K}_{\mathfrak{p}} \bullet=S_{\mathfrak{p}}^{-1} \mathscr{K}$ of Corollary 3.9. We know that $\mathrm{R}_{\mathscr{L}}^{\bullet} \cong\left(\mathrm{R}_{\mathscr{K}}^{\bullet}\right)_{\mathfrak{p}} \bullet$ is a local graded ring. Suppose that the maximal homogeneous ideal of $\mathrm{R}_{\mathscr{L}}^{\bullet}$ belongs to the image of $\rho_{\mathscr{L}}^{\bullet}$. Then $\mathfrak{p}^{\bullet}$ belongs to the image of $\rho_{\mathscr{K}}^{\bullet}$.

Proof By naturality of $\rho^{\bullet}$, the following diagram commutes:

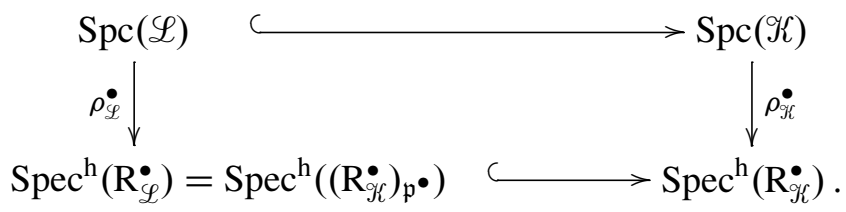

Since the lower map sends the maximal ideal of $\mathrm{R}_{\mathscr{L}}^{\bullet}$ to $\mathfrak{p}^{\bullet}$, we get the result.

The above can be combined with the following:

Proposition 7.2 Let $\mathscr{K}$ be a tensor triangulated category such that $\mathrm{R}_{\mathscr{K}}^{\bullet}$ is local with maximal homogeneous ideal $\mathfrak{m}^{\bullet}$. Then the following conditions are equivalent:

(i) There exists a prime $\mathscr{P} \in \operatorname{Spc}(\mathscr{K})$ such that $\rho_{\mathscr{K}}^{\bullet}(\mathscr{P})=\mathfrak{m}^{\bullet}$.

(ii) For every $n \geq 1$ and every homogeneous $f_{1}, \ldots, f_{n} \in \mathfrak{m}^{\bullet}$, the product of their cones cone $\left(f_{1}\right) \otimes \cdots \otimes \operatorname{cone}\left(f_{n}\right)$ is nonzero in $\mathscr{K}$.

Proof Suppose that $\rho_{\mathscr{\mathscr { K }}}^{\bullet}(\mathscr{P})=\mathrm{m}^{\bullet}$ and let $f_{1}, \ldots, f_{n} \in \mathrm{R}_{\mathscr{K}}^{\bullet}$ be homogeneous elements such that cone $\left(f_{1}\right) \otimes \cdots \otimes \operatorname{cone}\left(f_{n}\right)=0 \in \mathscr{P}$. Since $\mathscr{P}$ is prime, this implies cone $\left(f_{i}\right) \in$ $\mathscr{P}$ for some $1 \leq i \leq n$. By Definition 5.1 this means $f_{i} \notin \rho_{\mathscr{K}}^{\bullet}(\mathscr{P})$, hence $f_{i} \notin \mathfrak{m} \bullet$

Conversely, suppose that $\mathcal{S}:=\left\{\operatorname{cone}\left(f_{1}\right) \otimes \cdots \otimes \operatorname{cone}\left(f_{n}\right) \mid n \geq 1, f_{1}, \ldots, f_{n} \in\right.$ $\mathfrak{m}^{\bullet}$ homogeneous $\} \cup\{\mathbb{1}\}$ does not contain zero. This $\mathcal{S}$ is $\otimes$-multiplicative by construction. By Zorn, more precisely by [1, Lemma 2.2 applied to $\mathscr{F}=0$ ], there exists a prime $\mathscr{P} \in \operatorname{Spc}(\mathscr{K})$ such that $\mathscr{P} \cap \mathcal{S}=\varnothing$. Hence, for every homogeneous $f \in \mathfrak{m}^{\bullet}$, we have cone $(f) \in \mathcal{S}$, so cone $(f) \notin \mathscr{P}$, that is $f \in \rho_{\mathscr{K}}^{\bullet}(\mathscr{P})$, by Definition 5.1. We have shown $\mathfrak{m}^{\bullet} \subset \rho_{\mathscr{K}}^{\bullet}(\mathscr{P})$ and this is enough since $\mathfrak{m}^{\bullet}$ is maximal. 
We now turn to the situation where $\mathrm{R}_{\mathscr{K}}^{\bullet}$ is coherent. We shall say that a graded ring is coherent if every finitely generated homogeneous ideal is finitely presented. Of course, a noetherian ring is coherent.

For this part, we choose the invertible object $u=\Sigma(\mathbb{1})$. That is, we have

$$
\operatorname{Hom}_{\mathscr{K}}^{\bullet}(a, b)=\operatorname{Hom}_{\mathscr{K}}\left(a, \Sigma^{\bullet} b\right)
$$

for any $a, b \in \mathscr{K}$ and in particular, $\mathrm{R}_{\mathscr{K}}^{\bullet}=\operatorname{Hom}_{\mathscr{K}}(\mathbb{1}, \Sigma \bullet \mathbb{1})$.

Theorem 7.3 Let $\mathscr{Y}$ be a tensor triangulated category such that $\mathrm{R}_{\mathscr{K}}^{\bullet}$ is coherent (eg noetherian). Then the map $\rho_{\mathscr{K}}^{\bullet}: \operatorname{Spc}(\mathscr{K}) \rightarrow \operatorname{Spec}^{\mathrm{h}}\left(\mathrm{R}_{\mathscr{K}}^{\bullet}\right)$ is surjective.

We prove this result below. By surjectivity of $(-)^{0}: \operatorname{Spec}^{\mathrm{h}}\left(\mathrm{R}_{\mathscr{K}}^{\bullet}\right) \rightarrow \operatorname{Spec}\left(\mathrm{R}_{\mathscr{K}}\right)$ (see Remark 5.5) and since $\rho_{\mathscr{K}}=(-)^{0} \circ \rho_{\mathscr{K}}^{\bullet}$ (see Corollary 5.6 (b)), we immediately have:

Corollary 7.4 Let $\mathscr{K}$ be a tensor triangulated category such that $\mathrm{R}_{\mathscr{K}}^{\bullet}$ is coherent (eg noetherian). Then the map $\rho_{\mathscr{K}}: \operatorname{Spc}(\mathscr{K}) \rightarrow \operatorname{Spec}\left(\mathrm{R}_{\mathscr{K}}\right)$ is surjective.

The key application of the coherence assumption is the following proposition. Recall that a graded module is coherent if it is finitely presented and if any finitely generated graded submodule is finitely presented as well.

Proposition 7.5 Suppose that $\mathrm{R}_{\mathscr{K}}^{\bullet}$ is coherent (eg noetherian) and local, with maximal homogeneous ideal $\mathfrak{m}^{\bullet}$. Let $f \in \mathfrak{m}^{\bullet}$ be homogeneous and let $a \in \mathscr{K}$ be an object such that $\operatorname{Hom}_{\mathscr{K}}^{\bullet}(\mathbb{1}, a)$ is nonzero and coherent as graded $\mathrm{R}_{\mathscr{K}}^{\bullet}-$ module. Then $\operatorname{Hom}_{\mathscr{H}}^{\bullet}(\mathbb{1}, a \otimes \operatorname{cone}(f))$ is nonzero and coherent as well.

Proof Let $d \in \mathbb{Z}$ be the degree of $f$. We have a distinguished triangle

$$
a \stackrel{f \vdash a}{\longrightarrow} \Sigma^{d}(a) \longrightarrow a \otimes \operatorname{cone}(f) \longrightarrow \Sigma a
$$

which induces a long exact sequence under the homological functor $\operatorname{Hom}_{\mathscr{K}}(\mathbb{1},-)$. This long exact sequence can be wrapped up $(\oplus)$ into a periodic exact sequence

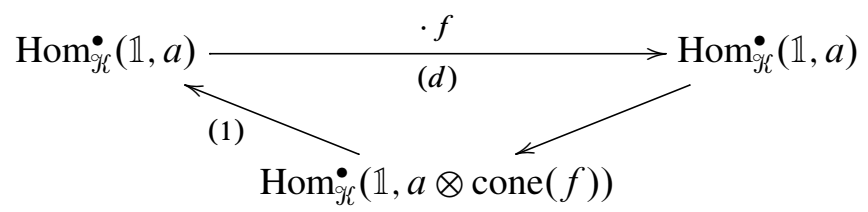

of graded $\mathrm{R}_{\mathscr{K}}^{\bullet}$-modules, where $(d)$ and (1) indicate homogeneous $\mathrm{R}_{\mathscr{K}}^{\bullet}$-linear homomorphisms of degree $d$ and 1 respectively. (Here we used our choice of $u=\Sigma(\mathbb{1})$.) If, ab absurdo, the lower module in (7-1) is zero, it means that $f$ acts surjectively on the coherent, hence finitely generated $\mathrm{R}_{\mathscr{K}}^{\bullet}-$ module $\operatorname{Hom}_{\mathscr{K}}^{\bullet}(\mathbb{1}, a)$. Since $f \in \mathfrak{m}^{\bullet}$, by the 
graded version of Nakayama's Lemma (see [20,1.10]), we would have $\operatorname{Hom}_{\mathscr{K}}^{\bullet}(\mathbb{1}, a)=0$, which is excluded. Hence the lower module is nonzero as claimed. The fact that it remains coherent is immediate: It fits in a short exact sequence with the cokernel and the kernel of the horizontal map $\cdot f$ of (7-1), which are both coherent. See [10, Proposition 1.2 and comments before Proposition 1.1].

Proof of Theorem 7.3 Since the localization of a coherent graded ring remains coherent (extend [12, Theorem 3] to graded-commutative), we can use Propositions 7.1 and 7.2 to assume that $\mathrm{R}_{\mathscr{K}}^{\bullet}$ is local and coherent and reduce the proof to showing that cone $\left(f_{1}\right) \otimes \cdots \otimes$ cone $\left(f_{n}\right) \neq 0$ for any homogeneous $f_{1}, \ldots, f_{n} \in \mathfrak{m}^{\bullet}$. By Proposition 7.5 , we even have the stronger fact that

$$
\operatorname{Hom}_{\mathscr{K}}\left(\mathbb{1}, \operatorname{cone}\left(f_{1}\right) \otimes \cdots \otimes \operatorname{cone}\left(f_{n}\right)\right) \neq 0
$$

by induction on $n \geq 1$. (Take of course $a=\mathbb{1}$ when $n=1$.)

We now prove surjectivity of $\rho_{\mathscr{K}}: \operatorname{Spc}(\mathscr{K}) \rightarrow \operatorname{Spec}\left(\mathrm{R}_{\mathscr{K}}\right)$ for another class of tensor triangulated categories.

Definition 7.6 Let us say that $\mathscr{K}$ is connective if $\operatorname{Hom}_{\mathscr{K}}\left(\mathbb{1}, \Sigma^{i}(\mathbb{1})\right)=0$ for all $i>0$.

Remark 7.7 Maybe surprisingly, this property is self-dual: If $\mathscr{K}$ is connective then $\mathscr{K}^{\text {op }}$ is connective as well. This comes from the fact that the suspension in $\mathscr{K}^{\mathrm{op}}$ must be $\Sigma^{-1}$. In other words, the results below cannot be applied merely under the "complementary" assumption that $\operatorname{Hom}_{\mathscr{K}}\left(\mathbb{1}, \Sigma^{i}(\mathbb{1})\right)=0$ for $i<0$, by claiming duality. Applying the following results to $\mathscr{K}^{\mathrm{op}}$ gives the same results.

As in Propositions 7.1 and 7.2, we have reductions to the local case:

Proposition 7.8 Let $\mathfrak{p} \in \operatorname{Spec}\left(\mathrm{R}_{\mathscr{K}}\right)$ be a prime. Consider the localization $\mathscr{L}:=\mathscr{K}_{\mathfrak{p}}$ of Corollary 3.11. We know that the ring $\mathrm{R}_{\mathscr{L}}=\left(\mathrm{R}_{\mathscr{K}}\right)_{\mathfrak{p}}$ is local. Suppose that the maximal ideal of $\mathrm{R}_{\mathscr{L}}$ belongs to the image of $\rho_{\mathscr{L}}$. Then $\mathfrak{p}$ belongs to the image of $\rho_{\mathscr{K}}$.

Proof Easy exercise on the naturality of $\rho$, as in the proof of Proposition 7.1.

Proposition 7.9 Let $\mathscr{K}$ be a tensor triangulated category whose central ring $\mathrm{R}_{\mathscr{K}}$ is local with maximal ideal $\mathfrak{m}$. Then the following conditions are equivalent:

(i) There exists a prime $\mathscr{P} \in \operatorname{Spc}(\mathscr{K})$ such that $\rho_{\mathscr{K}}(\mathscr{P})=\mathfrak{m}$.

(ii) For every $n \geq 1$ and every $f_{1}, \ldots, f_{n} \in \mathfrak{m}$, the product of their cones $\operatorname{cone}\left(f_{1}\right) \otimes$ $\cdots \otimes \operatorname{cone}\left(f_{n}\right)$ is nonzero in $\mathscr{K}$.

Proof Same proof as Proposition 7.2. 
Lemma 7.10 Let $\mathscr{K}$ be connective. Let $f_{1}, \ldots, f_{n} \in \mathrm{R}_{\mathscr{K}}$. Let $c=\operatorname{cone}\left(f_{1}\right) \otimes \cdots \otimes$ $\operatorname{cone}\left(f_{n}\right) \in \mathscr{K}$ be the product of their cones. Then, we have:

(a) $\operatorname{Hom}_{\varkappa}\left(\mathbb{1}, \Sigma^{i}(c)\right)=0$ for all $i>0$.

(b) There is a natural isomorphism $\mathbf{R}_{\mathscr{K}} /\left\langle f_{1}, \ldots, f_{n}\right\rangle \stackrel{\sim}{\rightarrow} \operatorname{Hom}_{\mathscr{K}}(\mathbb{1}, c)$.

Proof By induction on $n \geq 0$. For $n=0$, that is for $c=\mathbb{1}$, statement (a) is the connectivity assumption and (b) is trivial. For the induction step, let $n \geq 1$. Consider $d=\operatorname{cone}\left(f_{1}\right) \otimes \cdots \otimes \operatorname{cone}\left(f_{n-1}\right)$ and the distinguished triangle

$$
d \stackrel{\left(f_{n}\right) \vdash_{d}}{\longrightarrow} d \longrightarrow c \longrightarrow \Sigma(d)
$$

obtained by tensoring $d$ with the triangle defining cone $\left(f_{n}\right)$. Consider the long exact sequence obtained by applying $\operatorname{Hom}_{\mathscr{K}}(\mathbb{1},-)$ to the above triangle (and Proposition 2.2): $\cdots \rightarrow \operatorname{Hom}_{\mathscr{K}}\left(\mathbb{1}, \Sigma^{i} d\right) \stackrel{\cdot f_{n}}{\longrightarrow} \operatorname{Hom}_{\mathscr{K}}\left(\mathbb{1}, \Sigma^{i} d\right) \rightarrow \operatorname{Hom}_{\mathcal{K}^{\prime}}\left(\mathbb{1}, \Sigma^{i} c\right) \rightarrow \operatorname{Hom}_{\mathcal{K}}\left(\mathbb{1}, \Sigma^{i+1} d\right) \rightarrow \cdots$ for $i \in \mathbb{Z}$. The induction hypothesis (for $d$ ) applied to this long exact sequence around $i>0$ gives (a); applied around $i=0$, it gives (b).

Proposition 7.11 Let $\mathscr{K}$ be a connective tensor triangulated category and let $I \subsetneq \mathrm{R}_{\mathscr{K}}$ be a proper ideal. Then for any $f_{1}, \ldots, f_{n} \in I$ the product of their cones does not vanish: cone $\left(f_{1}\right) \otimes \cdots \otimes \operatorname{cone}\left(f_{n}\right) \neq 0$.

Proof By Lemma 7.10 (b), we have $\operatorname{Hom}_{\mathscr{K}}\left(\mathbb{1}\right.$, cone $\left.\left(f_{1}\right) \otimes \cdots \otimes \operatorname{cone}\left(f_{n}\right)\right) \neq 0$.

Corollary 7.12 Let $\mathscr{K}$ be a connective tensor triangulated category such that $\mathrm{R}_{\mathscr{K}}$ is local with maximal ideal $\mathfrak{m}$. Then there exists $\mathscr{P} \in \operatorname{Spc}(\mathscr{K})$ such that $\rho_{\mathscr{K}}(\mathscr{P})=\mathfrak{m}$.

Proof It suffices to check condition (ii) of Proposition 7.9 and this follows from Proposition 7.11 applied to $I=\mathfrak{m}$.

Theorem 7.13 Let $\mathscr{K}$ be a connective tensor triangulated category (Definition 7.6). Then the map $\rho_{\mathscr{K}}: \operatorname{Spc}(\mathscr{K}) \longrightarrow \operatorname{Spec}\left(\mathrm{R}_{\mathscr{K}}\right)$ is surjective.

Proof This is immediate from Proposition 7.8 and Corollary 7.12 once we know that $\mathscr{K}_{\mathfrak{p}}$ remains connective for every $\mathfrak{p} \in \operatorname{Spec}\left(\mathrm{R}_{\mathscr{K}}\right)$. This is obvious by construction of $\mathscr{K}_{\mathfrak{p}}=S_{\mathfrak{p}}^{-1} \mathscr{K}$ (see Corollary 3.11), since $S_{\mathfrak{p}}=\mathrm{R}_{\mathscr{K}} \backslash \mathfrak{p}$ sits in degree zero. 
Corollary 7.14 Let $\mathscr{K}$ be a connective tensor triangulated category such that $\mathrm{R}_{\mathscr{K}}^{<0}=$ $\bigoplus_{i<0} \operatorname{Hom}_{\mathscr{K}}\left(\mathbb{1}, \Sigma^{i} \mathbb{1}\right)$ is a nil-ideal. (In more topological notation, we are assuming that any element $f: \Sigma^{j} \mathbb{1} \rightarrow \mathbb{1}$ is zero for $j<0$ and nilpotent for $j>0$.) Then $\operatorname{Spec}^{\mathrm{h}}\left(\mathrm{R}_{\mathscr{K}}^{\bullet}\right) \cong \operatorname{Spec}\left(\mathrm{R}_{\mathscr{K}}\right)$ via $(-)^{0}$. In particular, $\rho_{\mathscr{K}}^{\bullet}: \operatorname{Spc}(\mathscr{K}) \rightarrow \operatorname{Spec}^{\mathrm{h}}\left(\mathrm{R}_{\mathscr{K}}^{\bullet}\right)$ coincides with $\rho_{\mathscr{K}}$ and is surjective.

Proof We have the commutative diagram of Corollary 5.6 (b) and we know by Theorem 7.13 that $\rho_{\mathscr{K}}$ is surjective. So, it suffices to show that $(-)^{0}: \operatorname{Spec}^{\mathrm{h}}\left(\mathrm{R}_{\mathscr{K}}^{\bullet}\right) \rightarrow \operatorname{Spec}\left(\mathrm{R}_{\mathscr{K}}\right)$, $\mathfrak{p}^{\bullet} \mapsto \mathfrak{p}^{0}$ is a bijection. Since $\mathrm{R}_{\mathscr{K}}^{<0}$ is a homogeneous nil-ideal, it belongs to all homogeneous prime ideals. Hence, we have $\mathfrak{p}^{\bullet}=\mathrm{R}_{\mathscr{K}}^{<0} \oplus \mathfrak{p}^{0}$ for all $\mathfrak{p}^{\bullet} \in \operatorname{Spec}^{\mathrm{h}}\left(\mathrm{R}_{\mathscr{K}}^{\bullet}\right)$ and the bijection follows easily.

\section{Some examples, old and new}

Proposition 8.1 Let $A$ be a commutative ring and let $\mathscr{K}=\mathrm{K}^{\mathrm{b}}(A-$ proj $)$ be the tensor triangulated category of perfect complexes. Then $\rho_{\mathscr{Y}}: \operatorname{Spc}\left(\mathrm{K}^{\mathrm{b}}(A-\operatorname{proj})\right) \rightarrow \operatorname{Spec}(A)$ is a homeomorphism, inverse to the one given in Example 4.4.

Proof Recall that the homeomorphism $\operatorname{Spec}(A) \stackrel{\sim}{\rightarrow} \operatorname{Spc}\left(\mathrm{K}^{\mathrm{b}}(A-\operatorname{proj})\right)$ is given by $\mathfrak{p} \mapsto \mathscr{P}(\mathfrak{p})=\left\{M_{\bullet} \in \mathrm{K}^{\mathrm{b}}(A-\operatorname{proj}) \mid\left(M_{\bullet}\right)_{\mathfrak{p}}=0\right.$ in $\left.\mathrm{K}^{\mathrm{b}}\left(A_{\mathfrak{p}}-\operatorname{proj}\right)\right\}$. Let $\mathfrak{p} \in \operatorname{Spec}(A)$. We need to show that $\rho_{\mathscr{K}}(\mathscr{P}(\mathfrak{p}))=\mathfrak{p}$. This is easy: For $f: \mathbb{1} \rightarrow \mathbb{1}$, ie for $f \in A$, we have by definition of $\rho_{\mathscr{K}}$ in $(5-1)$ that $f \in \rho_{\mathscr{K}}(\mathscr{P}(\mathfrak{p})) \Leftrightarrow \operatorname{cone}(f) \notin \mathscr{P}(\mathfrak{p}) \Leftrightarrow$ the image of $f: \mathbb{1} \rightarrow \mathbb{1}$ is not invertible in $\mathrm{K}^{\mathrm{b}}\left(A_{\mathfrak{p}}\right.$-proj $) \Leftrightarrow$ the image of $f$ in $A_{\mathfrak{p}}$ is not invertible $\Leftrightarrow f \in \mathfrak{p}$.

Remark 8.2 For a nonaffine scheme $X$, this result fails for $\mathscr{K}=\mathrm{D}^{\text {perf }}(X)$, simply because there aren't always enough global sections, as can be checked for $X=\mathbb{P}_{k}^{n}$. However, in this case, or more generally if $X=\operatorname{Proj}\left(A^{\bullet}\right)$ is a projective scheme with $A^{\bullet}$ such that the natural map $A^{d} \rightarrow \Gamma(X, \mathcal{O}(d))$ is an isomorphism for large $d$ (see for instance [13, Exercise 2.5.9, page 125]) and such that $A^{0}=\Gamma\left(X, \mathcal{O}_{X}\right)$, then $\operatorname{Spec}^{\mathrm{h}}\left(A^{\bullet}\right)=\operatorname{Spec}^{\mathrm{h}}(\Gamma(X, \mathcal{O}(\bullet)))$. In this case, we can choose $u=\mathcal{O}(1)$ as invertible object in $\mathscr{K}$ and the map $\rho_{\mathscr{K}}^{\bullet}: \operatorname{Spc}\left(\mathrm{D}^{\text {perf }}(X)\right) \longrightarrow \operatorname{Spec}^{\mathrm{h}}\left(A^{\bullet}\right)$ induces a homeomorphism onto $X$. This is similar to the above, using [1, Corollary 5.6], and is left to the reader.

I'm thankful to C Walter for the following example of nonsurjectivity of $\rho_{\mathscr{K}}$.

Example 8.3 Let $(A, \mathfrak{m})$ be a regular local ring and $U=\operatorname{Spec}(A) \backslash\{\mathfrak{m}\}$ be its punctured spectrum. Let $\mathscr{K}=\mathrm{D}^{\text {perf }}(U)$. When $d=\operatorname{dim}_{\text {Krull }}(A) \geq 2$, we have $\mathrm{R}_{\mathscr{K}}=$ $A$ and $\operatorname{Spec}\left(\operatorname{R}_{\mathscr{K}}\right)=\operatorname{Spec}(A)$ which is strictly bigger than $U=\operatorname{Im}\left(\rho_{\mathscr{K}}\right)$. In fact, 
$\mathrm{R}_{\mathscr{K}}^{\bullet} \simeq A \oplus \mathrm{R}_{\mathscr{K}}^{d-1}$ where $\mathrm{R}_{\mathscr{K}}^{d-1}=H^{d-1}\left(U, \mathcal{O}_{U}\right)$ is an injective envelope of the residue field $A / \mathfrak{m}$. This ring $\mathrm{R}_{\mathscr{K}}^{\bullet}$ is neither connective nor coherent.

Remark 8.4 The above proof of Proposition 8.1 uses the classification of thick triangulated $\otimes$-ideals, hidden in [1, Corollary 5.6]. Yet our Theorem 7.13 proves surjectivity of $\rho_{\mathscr{K}}: \operatorname{Spc}(\mathscr{K}) \longrightarrow \operatorname{Spec}(A)$ without the classification. It is therefore interesting to find a direct proof of injectivity, for this would yield a new proof of classification, via [1, Theorem 4.10]. Actually such direct proofs of the injectivity of $\rho_{\mathscr{K}}$ do exist in this case. There is a rather elementary such proof, using only perfect complexes, but it is a little long to include here. Another proof, due to Amnon Neeman, uses bigger categories but can be sketched as follows. The general machinery developed above allows us to reduce to the case of $(A, \mathfrak{m})$ local and to show that the preimage in $\operatorname{Spc}(\mathscr{K})$ of the maximal ideal consists only of the ideal zero. To do that, it suffices to show that for any nonzero object $X \in \mathscr{K}=\mathrm{D}^{\text {perf }}(A)$ there exists $f_{1}, \ldots, f_{r} \in \mathfrak{m}$ such that $\operatorname{Kos}(f) \in\langle X\rangle$, where $\operatorname{Kos}(f):=\operatorname{cone}\left(f_{1}\right) \otimes \cdots \otimes \operatorname{cone}\left(f_{r}\right)$. One can reduce to $A$ noetherian local. Then in the big derived category $\mathrm{D}(A)$, the complex $X \otimes^{L} \kappa$ is a finite sum of shifts of the residue field $\kappa:=A / \mathfrak{m}$. Then, using the $t$-structure on $\mathrm{D}(A)$, one can show by induction that any bounded complex with finite length homology belongs to $\langle\kappa\rangle$ and hence to the localizing triangulated subcategory $\langle X\rangle_{\text {loc }}$ generated by $X$ in $\mathrm{D}(A)$. In particular, if $f=\left(f_{1}, \ldots, f_{r}\right)$ is a sequence generating $\mathfrak{m}$, we have $\operatorname{Kos}(f) \in\langle X\rangle_{\text {loc }} \cap \mathscr{K}$. Since the object $X \in \mathscr{K}=(\mathrm{D}(A))^{\mathrm{c}}$ is compact in the compactly generated $\mathrm{D}(A)$, we can use a general result of triangulated categories (see Neeman [27, Theorem 4.4.9]) which implies here that $\langle X\rangle_{\text {loc }} \cap \mathscr{K}=\langle X\rangle$ and we get $\operatorname{Kos}(f) \in\langle X\rangle$ as wanted.

Let us switch to modular representation theory. The following result is new although known to some experts (announced for instance in talks by Julia Pevtsova, joint work with Paul Smith; see also Benson et al [8]):

Proposition 8.5 Let $G$ be a finite group (scheme) and $k$ be a field. Consider $\mathscr{K}=$ $\mathrm{D}^{\mathrm{b}}\left(k G\right.$-mod) with $\otimes=\otimes_{k}$. Its graded central ring $\mathrm{R}_{\mathscr{\kappa}}^{\bullet}$ (Definition 3.2) is $\mathrm{H}^{\bullet}(G, k)$ and the map $\rho_{\mathscr{K}}^{\bullet}: \operatorname{Spc}\left(\mathrm{D}^{\mathrm{b}}(k G-\bmod )\right) \rightarrow \operatorname{Spec}^{\mathrm{h}}\left(\mathrm{H}^{\bullet}(G, k)\right)$ is a homeomorphism.

Proof Let $\mathscr{F}=\mathrm{K}^{\mathrm{b}}(k G$-proj $) \subset \mathscr{K}$. By Rickard [30], the quotient $\mathscr{K} / \mathscr{F}$ is equivalent to the stable category $k G-$ stab, whose spectrum is described in [1, Corollary 5.10] as

$$
\begin{aligned}
\varphi: \operatorname{Proj}\left(\mathrm{H}^{\bullet}(G, k)\right) & \stackrel{\sim}{\longrightarrow} \operatorname{Spc}(k G-\mathrm{stab}) \\
\mathfrak{p}^{\bullet} & \longmapsto \mathscr{P}\left(\mathfrak{p}^{\bullet}\right):=\left\{M \mid \operatorname{Ann}_{\mathrm{H}^{\bullet}(G, k)}\left(\mathrm{H}^{\bullet}(G, M)\right) \not \subset \mathfrak{p}^{\bullet}\right\} .
\end{aligned}
$$


We then have the following picture:

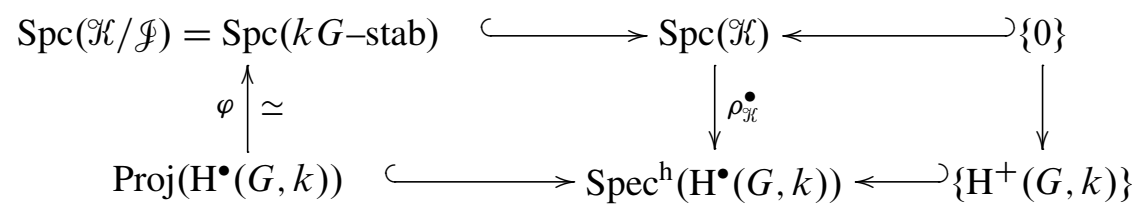

We claim that this diagram commutes. Indeed, the graded $\operatorname{ring} \mathrm{H}^{\bullet}(G, k)$ is local with maximal ideal $\mathrm{H}^{+}(G, k)$. We claim that $\mathscr{K}$ is local as well. This is obvious since we have a forgetful $\otimes$-functor $\mathscr{K} \rightarrow \mathrm{D}^{\mathrm{b}}(k)$ whose kernel is zero (exactness of a complex of $k G$-modules being a property of the underlying complex of vector spaces). So $0 \in$ $\operatorname{Spc}(\mathscr{K})$ and $\mathscr{K}$ is local by Proposition 4.2. It is obvious that $\rho_{\mathscr{K}}^{\bullet}(0)=\mathrm{H}^{+}(G, k)$ since no homogeneous element of positive degree can be invertible. Then the right-hand square of the above diagram commutes. To check that the left-hand square also commutes, consider a homogeneous prime $\mathfrak{p}^{\bullet} \in \operatorname{Proj}\left(\mathrm{H}^{\bullet}(G, k)\right)$ and a homogeneous element $f \in \mathrm{H}^{d}(G, k)$ for some $d \geq 0$. Let $q: \mathscr{K} \longrightarrow k G-$ stab be the localization functor. Then, $f \in \rho_{\mathscr{K}}^{\bullet}\left(q^{-1}\left(\mathscr{P}\left(\mathfrak{p}^{\bullet}\right)\right)\right) \Leftrightarrow \operatorname{cone}(f) \notin q^{-1}\left(\mathscr{P}\left(\mathfrak{p}^{\bullet}\right)\right) \Leftrightarrow q(\operatorname{cone}(f)) \notin \mathscr{P}\left(\mathfrak{p}^{\bullet}\right) \Leftrightarrow$ $\operatorname{Ann}_{\mathrm{H}^{\bullet}(G, k)}\left(\mathrm{H}^{\bullet}(G, q(\operatorname{cone}(f)))\right) \subset \mathfrak{p}^{\bullet} \Leftrightarrow \mathfrak{p}^{\bullet} \in V\left(\operatorname{Ann}_{\mathrm{H}^{\bullet}(G, k)}\left(\mathrm{H}^{\bullet}(G, q(\operatorname{cone}(f)))\right)\right)=$ $V(f)$. The last equality comes from the fact that $q(\operatorname{cone}(f))=\operatorname{cone}(q(f))$ is the suspension of the so-called Carlson module $L_{f}$, whose support is known to be $V(f)$; see [7, Proposition II.5.9.1, page 186]. In short, we have $f \in \rho_{\mathscr{\mathscr { K }}}^{\bullet}\left(q^{-1}\left(\mathscr{P}\left(\mathfrak{p}^{\bullet}\right)\right)\right) \Leftrightarrow$ $f \in \mathfrak{p}^{\bullet}$, which proves $\rho_{\mathscr{K}}^{\bullet}\left(q^{-1}\left(\varphi\left(\mathfrak{p}^{\bullet}\right)\right)\right)=\mathfrak{p}^{\bullet}$, as wanted.

Finally, we claim that $\operatorname{Spc}(\mathscr{K})=\operatorname{Spc}(\mathscr{K} / \mathscr{F}) \cup\{0\}$. This follows from the fact that every nonzero $\otimes$-ideal $\mathscr{P} \subset \mathscr{K}$ contains $k G$. Indeed, for any $M_{\bullet} \in \mathrm{D}^{\mathrm{b}}(k G-$ mod $)$, we have by Frobenius reciprocity that $k G \otimes M_{\bullet} \simeq \operatorname{ind}_{e}^{G} \circ \operatorname{res}_{e}^{G}\left(M_{\bullet}\right)$ and since $\operatorname{res}_{e}^{G}\left(M_{\bullet}\right) \in$ $\mathrm{D}^{\mathrm{b}}(k-\mathrm{mod})$, the complex $\operatorname{res}_{e}^{G}\left(M_{\bullet}\right)$ is isomorphic to a complex of vector spaces with zero differentials. So, if $M_{\bullet} \neq 0$ then $k G \otimes M_{\bullet}$ has a direct summand isomorphic to $k G$. Hence if $0 \neq \mathscr{P} \in \operatorname{Spc}(\mathscr{K})$ then $\mathscr{E}=\mathrm{K}^{\mathrm{b}}(k G$-proj $)=\langle k G\rangle \subset \mathscr{P}$, that is, $\mathscr{P}$ belongs to the image of $\operatorname{Spc}(\mathscr{K} / \mathscr{F}) \hookrightarrow \operatorname{Spc}(\mathscr{K})$; see Remark 1.4.

We have shown that the above diagram commutes, that the top row describes a partition $\operatorname{Spc}(\mathscr{K})=\operatorname{Spc}(\mathscr{K} / \mathscr{F}) \cup\{0\}$ formed by the closed point $\{0\}$ and its open complement $\operatorname{Spc}(\mathscr{K} / \mathscr{\mathscr { L }})$. Since $\rho_{\mathscr{K}}^{\bullet}$ is a bijection on both parts, we see that $\rho_{\mathscr{K}}^{\bullet}$ is bijective. Since $\rho_{\mathscr{K}}^{\bullet}$ is continuous, it only remains to check that it is open. This is very easy. The only open subset of $\operatorname{Spc}(\mathscr{K})$ which contains $\{0\}$ is the whole $\operatorname{Spc}(\mathscr{K})$. Any other open of $\operatorname{Spc}(\mathscr{K})$ will be in $\operatorname{Spc}(\mathscr{K} / \mathscr{F})$, hence will have an open image in $\operatorname{Proj}\left(\mathrm{H}^{\bullet}(G, k)\right)$, which is itself open in $\operatorname{Spec}^{\mathrm{h}}\left(\mathrm{H}^{\bullet}(G, k)\right)$.

Remark 8.6 In this example of $\mathscr{K}=\mathrm{D}^{\mathrm{b}}\left(k G\right.$-mod), like for the case of $\mathrm{K}^{\mathrm{b}}(A-$ proj $)$, the general results of Section 7 actually provide a proof of the surjectivity of the 
$\operatorname{map} \rho_{\mathscr{K}}^{\bullet}: \operatorname{Spc}\left(\mathrm{D}^{\mathrm{b}}(k G-\bmod )\right) \longrightarrow \operatorname{Spec}^{\mathrm{h}}\left(\mathrm{H}^{\bullet}(G, k)\right)$ without using classification results. Indeed, $\mathrm{H}^{\bullet}(G, k)$ is noetherian by the Venkov-Evens Theorem (see [7, Theorem II.4.2.1, page 126]) so we can apply Theorem 7.3. It would be interesting to prove injectivity directly, in the spirit of Remark 8.4. Again, such a direct proof would provide a new proof of classification, via general tensor triangular theory [1, Theorem 4.10].

Section 7 also applies to the following more analytic example.

Example 8.7 Let $G$ be a compact (Lie) group and $K K^{G}$ the tensor triangulated category which has separable $G-C^{*}$-algebras as objects, equivariant Kasparov's $K K-$ theory as morphisms and tensor given by the minimal tensor product with diagonal $G$-action. Details can be found in Meyer's survey [23] or in Dell'Ambrogio's thesis [11, Chapter 5]. Here, the ring $\mathrm{R}_{K K^{G}}=K K_{0}^{G}(\mathbb{C}, \mathbb{C})=K_{0}^{G}(\mathbb{C})=: R(G)$ is the Grothendieck group of continuous complex representations of $G$, following Segal [31]. Dell'Ambrogio proves by other techniques that $\rho_{\mathscr{K}} G: \operatorname{Spc}\left(\mathscr{K}_{K} G\right) \rightarrow \operatorname{Spec}(R(G))$ is split surjective when $G$ is finite and where $\mathscr{K}^{G}$ is the triangulated subcategory of $K K^{G}$ generated by the unit. He also proves that the map $\rho_{\mathscr{K}} G: \operatorname{Spc}\left(\mathscr{K}_{K} G\right) \rightarrow \operatorname{Spec}(R(G))$ is a homeomorphism for $G$ trivial and conjectures that this holds for all finite groups. With Corollary 7.4 we can generalize the surjectivity from finite groups to any compact group.

Corollary 8.8 Let $G$ be a compact Lie group. With the above notation, the continuous map $\rho_{K K^{G}}: \operatorname{Spc}\left(K K^{G}\right) \rightarrow \operatorname{Spec}(R(G))$ is surjective. Consequently, so is $\rho_{\mathscr{K}} G: \operatorname{Spc}\left(\mathscr{K}^{G}\right) \rightarrow \operatorname{Spec}(R(G))$.

Proof By Bott periodicity, $\operatorname{Spec}^{\mathrm{h}}\left(\mathrm{R}_{K K^{G}}^{\bullet}\right)=\operatorname{Spec}\left(\mathrm{R}_{K K^{G}}\right)$. In addition, $K K_{1}^{G}(\mathbb{C}, \mathbb{C})=$ $K_{1}^{G}(\mathbb{C})=R(G) \otimes K_{1}(\mathbb{C})=0$; see [28, Remark 2.8.5]. So, $\mathrm{R}_{K K^{G}}^{i}=0$ for $i$ odd. It is a result of Segal [31, Corollary 3.3] that $R(G)$ is noetherian for any compact Lie group and consequently $\mathrm{R}_{K K^{G}}$ is noetherian as well. Corollary 7.4 then gives the result. The last statement follows since $\rho_{K K^{G}}$ factors via $\rho_{\mathscr{K}_{K} G}$, using ${ }_{K_{K}} G \hookrightarrow K K^{G}$.

\section{Spectra of topological spectra}

Let us consider the tensor triangulated category $\mathscr{K}=\mathrm{SH}^{\mathrm{fin}}$, the topological stable homotopy category of finite spectra; see Margolis [22]. The tensor product is induced by the smash product and the unit $\mathbb{1}=S^{0}$ is the sphere spectrum. We have $\mathrm{R}_{\mathscr{K}}=\mathbb{Z}$ and $\mathrm{R}_{\mathscr{K}}^{\bullet}$ is connective with nilpotent $\mathrm{R}_{\mathscr{K}}^{<0}$. So, by Corollary 7.14, we can focus on $\rho_{\mathscr{K}}$.

Let us denote the set of prime numbers by $\mathbb{P} \subset \mathbb{N}=\{0,1,2,3, \ldots\}$. Let $p \in \mathbb{P}$ and let $\mathrm{SH}_{(p)}^{\mathrm{fin}}$ be the localization of $\mathrm{SH}^{\mathrm{fin}}$ at $p$, as in Corollary 3.11 applied to $\mathfrak{p}=p \mathbb{Z}$. 
We recall the following major result from Hopkins and Smith [15]; see also Ravenel [29].

Theorem 9.1 (Hopkins-Smith) Let $p \in \mathbb{P}$ be a prime. Recall that Morava $K-$ theories define a collection of functors $K_{p, n}: \mathrm{SH}_{(p)}^{\mathrm{fin}} \longrightarrow \mathscr{V}_{p, n}$ into $\otimes$-categories $\mathscr{V}_{p, n}$ of free graded modules, with index $n \in \mathbb{N}$. All functors $K_{p, n}$ satisfy a Künneth formula, ie they are $\otimes$-functors. Let $\mathscr{C}_{p, n}:=\operatorname{Ker}\left(K_{p, n}\right)$. Then,

$$
0=: \mathscr{C}_{p, \infty} \subsetneq \cdots \subsetneq \mathscr{C}_{p, n} \subsetneq \mathscr{C}_{p, n-1} \subsetneq \cdots \subsetneq \mathscr{C}_{p, 1} \subsetneq \mathscr{C}_{p, 0} \subsetneq \mathrm{SH}_{(p)}^{\mathrm{fin}}
$$

are all the thick triangulated $\otimes$-ideals of $\mathrm{SH}_{(p)}^{\mathrm{fin}}$. Moreover, $\bigcap_{n \in \mathbb{N}} \mathscr{C}_{p, n}=0$.

Corollary 9.2 Every proper thick triangulated $\otimes-i d e a l$ of $\mathrm{SH}_{(p)}^{\mathrm{fin}}$ is prime. In particular, $\mathrm{SH}_{(p)}^{\mathrm{fin}}$ is a local tensor triangulated category (see Definition 4.1).

Proof Indeed, since $K_{p, n}$ satisfies a Künneth formula and since the graded tensor product of two free graded modules cannot vanish unless one of the factors is zero, $\operatorname{Ker}\left(K_{p, n}\right)$ is prime for every $n \geq 0$. Hence so is their intersection $\mathscr{C}_{p, \infty}=0$. Then $\mathrm{SH}_{(p)}^{\text {fin }}$ is local by Proposition 7.8 (iii).

Example 9.3 For $n=0$, the Morava $K$-theory $K_{p, 0}$ is rational (singular) homology: $K_{p, 0}=H_{*}(-, \mathbb{Q})$ and $\mathscr{C}_{p, 0}$ is therefore the subcategory of torsion finite spectra localized at $p$. Note that the preimage of $\mathscr{C}_{p, 0}$ in $\mathrm{SH}^{\mathrm{fin}}$ under the localization functor $\mathrm{SH}^{\mathrm{fin}} \longrightarrow \mathrm{SH}_{(p)}^{\mathrm{fin}}$ is the subcategory of torsion finite spectra, $\mathrm{SH}_{\mathrm{tor}}^{\mathrm{fin}}$, that is, the kernel of rational homology. In particular, it is independent of $p$.

Proposition 9.4 Let $p \in \mathbb{P}$ be a prime. The central ring $\mathrm{R}_{\mathrm{SH}_{(p)}^{\mathrm{fin}}}=\mathbb{Z}_{(p)}$ is the ring of integers localized at $p$. The map $\rho:=\rho_{\mathrm{SH}_{(p)}^{\mathrm{fin}}}: \operatorname{Spc}\left(\mathrm{SH}_{(p)}^{\mathrm{fin}}\right) \longrightarrow \operatorname{Spec}\left(\mathbb{Z}_{(p)}\right) \operatorname{maps} \mathscr{C}_{p, n}$ to the maximal ideal $p \mathbb{Z}_{(p)}$ for every $n>0$ and maps $\mathscr{C}_{p, 0}$ to 0 .

Proof By definition (see (5-1)), the prime ideal $\rho\left(\mathscr{C}_{p, 0}\right)$ consists of all $f \in \mathrm{R}_{\mathrm{SH}_{(p)}^{\text {fin }}}=$ $\mathbb{Z}_{(p)}$ whose cone does not belong to $\mathscr{C}_{p, 0}$, ie is not torsion. So, $\rho\left(\mathscr{C}_{p, 0}\right)=\left\{f \in \mathbb{Z}_{(p)} \mid\right.$ $f$ is not an isomorphism rationally $\}=0$.

As $\rho$ is inclusion-reversing (Remark 5.2), and as $p \mathbb{Z}_{(p)}$ is maximal, in order to prove $\rho\left(\mathscr{C}_{p, n}\right)=p \mathbb{Z}_{(p)}$ for all $n \geq 1$, it suffices to prove it for $n=1$. The Morava $K$-theory $K_{p, 1}$ is a direct summand of $\bmod p$ topological $K$-theory. Hence $K_{p, 1}\left(S^{0} \stackrel{p}{\rightarrow} S^{0}\right)$ is the zero map. Therefore, $K_{p, 1}(\operatorname{cone}(p))=\mathbb{1} \oplus \mathbb{1}(1)$ in $\mathscr{V}_{p, 1}$ and in any case it is not zero. So cone $(p) \notin \operatorname{Ker}\left(K_{p, 1}\right)=\mathscr{C}_{p, 1}$, ie $p \in \rho\left(\mathscr{C}_{p, 1}\right)$ by definition; see (5-1). Thus $p \mathbb{Z}_{(p)} \subset \rho\left(\mathscr{C}_{p, 1}\right)$ and we have equality since $p \mathbb{Z}_{(p)}$ is the maximal ideal. 
Corollary 9.5 The spectrum of $\mathrm{SH}^{\mathrm{fin}}$ is the following topological space. We also describe the surjective continuous map $\rho:=\rho_{\mathrm{SH}^{\mathrm{fin}}}: \operatorname{Spc}\left(\mathrm{SH}^{\mathrm{fin}}\right) \longrightarrow \operatorname{Spec}(\mathbb{Z})$.

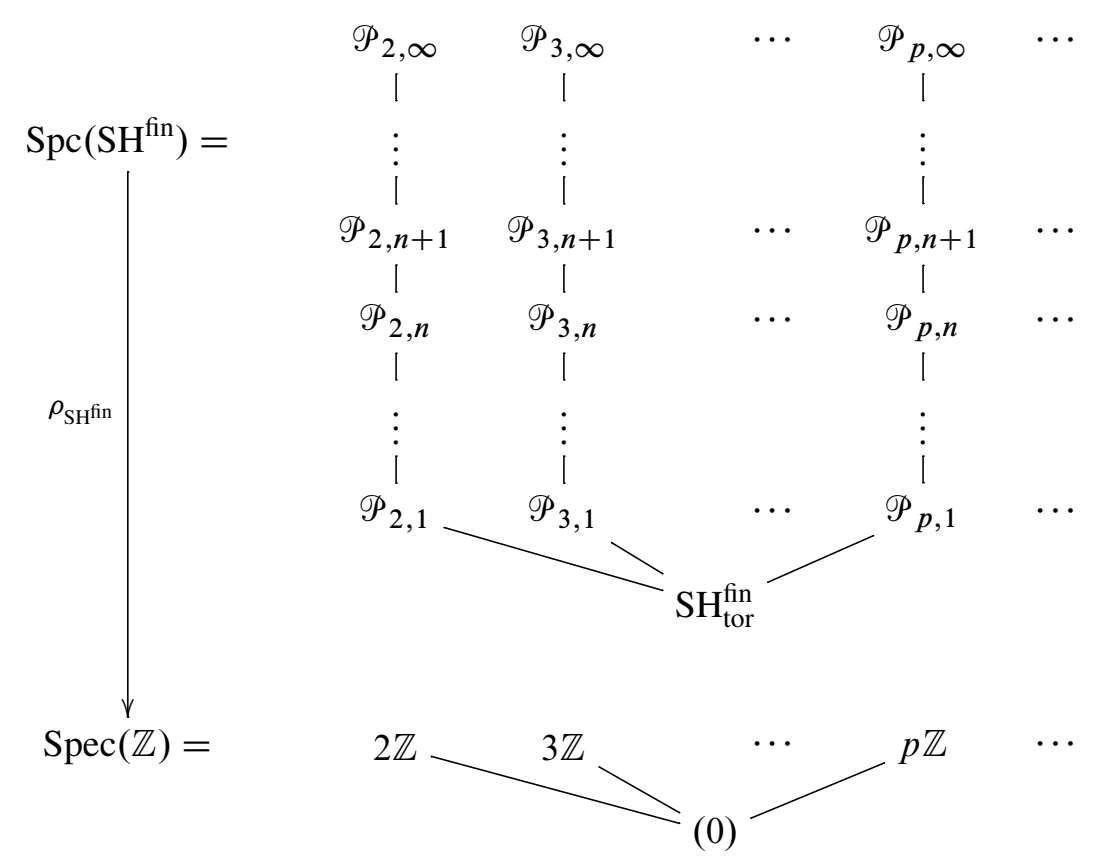

In the above picture, a line indicates that the higher prime is in the closure of the lower one (specialization). We have more precisely:

(a) The preimage of the dense point of $\operatorname{Spec}(\mathbb{Z})$ has only one point, $\rho^{-1}(0)=$ $\left\{\mathrm{SH}_{\mathrm{tor}}^{\mathrm{fin}}\right\}$, namely the subcategory of torsion spectra $\mathrm{SH}_{\mathrm{tor}}^{\mathrm{fin}}=\operatorname{Ker}\left(H_{*}(-, \mathbb{Q})\right)$.

(b) For each prime $p \in \mathbb{P}$ the preimage $\rho^{-1}(p \mathbb{Z})$ consists of the countable collection of $\mathscr{P}_{p, n}:=q_{p}^{-1}\left(\mathscr{C}_{p, n}\right)$ where $q_{p}: \mathrm{SH}^{\mathrm{fin}} \rightarrow \mathrm{SH}_{(p)}^{\mathrm{fin}}$ is the localization functor and where $1 \leq n \leq \infty$. When $n<\infty$, we have $\mathscr{P}_{p, n}=\operatorname{Ker}\left(K_{p, n} \circ q_{p}\right)=\left\{x \in \mathrm{SH}^{\mathrm{fin}} \mid\right.$ $\left.K_{p, n}\left(q_{p}(x)\right)=0\right\}$, where $K_{p, n}$ is the $n$-th Morava $K$-theory at $p$. Finally, $\mathscr{P}_{p, \infty}=\operatorname{Ker}\left(q_{p}\right)=\left\{x \in \mathrm{SH}^{\mathrm{fin}} \mid q_{p}(x)=0\right.$ in $\left.\mathrm{SH}_{(p)}^{\mathrm{fin}}\right\}$.

(c) $\mathrm{SH}_{\text {tor }}^{\mathrm{fin}}$ is the unique dense point of $\mathrm{Spc}\left(\mathrm{SH}^{\mathrm{fin}}\right)$. For each $p \in \mathbb{P}$ and each $1 \leq n \leq \infty$, the closure of $\left\{\mathscr{P}_{p, n}\right\}$ is $\overline{\left\{\mathscr{P}_{p, n}\right\}}=\left\{\mathscr{P}_{p, m} \mid n \leq m \leq \infty\right\}$. The closed points of $\operatorname{Spc}\left(\mathrm{SH}^{\text {fin }}\right)$ are exactly the $\mathscr{P}_{p, \infty}=\operatorname{Ker}\left(q_{p}\right)$ for all $p \in \mathbb{P}$.

(d) For an object $x \in \mathrm{SH}^{\text {fin }}$, we have $\operatorname{supp}(x)=\mathrm{Spc}\left(\mathrm{SH}^{\text {fin }}\right)$ if and only if $x$ is not torsion. When $x \in \mathrm{SH}_{\text {tor }}^{\mathrm{fin}}$, then

$$
\operatorname{supp}(x)=\bigcup_{\substack{p \in \mathbb{P}, q_{p}(x) \neq 0}} \overline{\left\{\mathscr{P}_{p, n(x, p)}\right\}}
$$


where the union involves only finitely many $p \in \mathbb{P}$ and where the integer $1 \leq$ $n(x, p)<\infty$, sometimes called the "type" of $q_{p}(x)$ (see [29, Definition 1.5.3]), is the smallest integer $n$ such that $q_{p}(x) \notin \mathscr{P}_{p, n}$.

(e) The proper nonempty closed subsets of $\mathrm{Spc}\left(\mathrm{SH}^{\mathrm{fin}}\right)$ are all possible finite unions of the following subsets: $\overline{\left\{\mathscr{P}_{p, n}\right\}}=\left\{\mathscr{P}_{p, m} \mid n \leq m \leq \infty\right\}$ for all $p \in \mathbb{P}$ and all $1 \leq n \leq \infty$.

Proof By Theorem 7.13, the map $\rho: \operatorname{Spc}\left(\mathrm{SH}^{\mathrm{fin}}\right) \rightarrow \operatorname{Spec}(\mathbb{Z})$ is surjective. So, to describe the set $\operatorname{Spc}\left(\mathrm{SH}^{\mathrm{fin}}\right)$, it suffices to describe the preimages $\rho^{-1}(0)$ and $\rho^{-1}(p \mathbb{Z})$ for $p \in \mathbb{P}$. Let us start with the preimage of the generic point $(0) \in \operatorname{Spec}(\mathbb{Z})$. By Corollary 5.6 (c) for $S=\mathbb{Z} \backslash(0)$, the preimage of (0) is the image of $\operatorname{Spc}\left(\mathrm{SH}_{(0)}^{\mathrm{fin}}\right)$ in $\operatorname{Spc}\left(\mathrm{SH}^{\mathrm{fin}}\right)$, that is, the image of $\operatorname{Spec}\left(q_{0}: \mathrm{SH}^{\mathrm{fin}} \rightarrow \mathrm{SH}_{(0)}^{\mathrm{fin}}\right)$. $\mathrm{But} \mathrm{SH}_{(0)}^{\mathrm{fin}}=\mathrm{SH}^{\mathrm{fin}} \otimes \mathbb{Q}$ is just $\mathrm{D}^{\mathrm{b}}(\mathbb{Q})$ and $q_{0}: \mathrm{SH}^{\mathrm{fin}} \rightarrow \mathrm{SH}^{\mathrm{fin}} \otimes \mathbb{Q} \simeq \mathrm{D}^{\mathrm{b}}(\mathbb{Q})$ is (total) rational homology (see [22, Theorem 8.1 .7 , page 113$])$. Since $D^{\mathrm{b}}(\mathbb{Q})$ has only $\{0\}$ as prime ideal, the preimage $\rho^{-1}(0)$ consists only of $\operatorname{Ker}\left(q_{0}\right)=\mathrm{SH}_{\text {tor }}^{\text {fin }}$. This proves (a).

Let now $p \in \mathbb{P}$ be a prime. By Corollary 5.6 (c) again but this time for $S=\mathbb{Z} \backslash p \mathbb{Z}$, we see that the fiber above $p \mathbb{Z}$ is the image in $\operatorname{Spc}\left(\mathrm{SH}^{\mathrm{fin}}\right)$ of the part of $\operatorname{Spc}\left(\mathrm{SH}_{(p)}^{\mathrm{fin}}\right)$ which maps to $p \mathbb{Z}_{(p)}$ under $\rho_{\mathrm{SH}_{(p)}^{\mathrm{fin}}}$. By the Hopkins and Smith's Theorem 9.1 and by Proposition 9.4, we know that this fiber is exactly the collection $\mathscr{C}_{p, n}$ for $1 \leq n \leq \infty$, whose images in $\mathrm{SH}^{\text {fin }}$ are the announced $\mathscr{P}_{p, n}$ for $1 \leq n \leq \infty$. This proves (b).

The inclusion $\mathscr{P}_{p, n} \subset \mathrm{SH}_{\text {tor }}^{\mathrm{fin}}$ is immediate from $\mathrm{SH}_{\text {tor }}^{\mathrm{fin}}=q_{p}^{-1}\left(\mathscr{C}_{p, 0}\right)$ and from the inclusions $\mathscr{C}_{p, n} \subset \mathscr{C}_{p, 0}$. Then (c) is easy to check from the definitions, recalling that in any tensor triangulated category $\mathscr{K}$, the closure of a point $\mathscr{P} \in \operatorname{Spc}(\mathscr{K})$ is described by $\overline{\{\mathscr{P}\}}=\{\mathscr{Q} \in \operatorname{Spc}(\mathscr{K}) \mid \mathscr{Q} \subset \mathscr{P}\}$; see [1, Proposition 2.9].

Now, let $x \in \mathrm{SH}^{\mathrm{fin}}$. If $x$ is not torsion, ie $x \notin \mathrm{SH}_{\mathrm{tor}}^{\mathrm{fin}}$, then for every $p \in \mathbb{P}$ and $n \in \mathbb{N} \cup\{\infty\}$ we have $x \notin \mathscr{P}_{p, n}$ as well, since $\mathscr{P}_{p, n} \subset \mathrm{SH}_{\text {tor }}^{\text {fin }}$. Hence by Definition 1.3 , we have $\operatorname{supp}(x):=\{\mathscr{P} \mid x \notin \mathscr{P}\}=\operatorname{Spc}\left(\mathrm{SH}^{\mathrm{fin}}\right)$. On the other hand, if $x$ is torsion, then it is well-known that $q_{p}(x) \neq 0$ for only finitely many $p \in \mathbb{P}$. (By Proposition 3.7, there exists $m \in \mathbb{Z}, m \neq 0$, such that $m l_{x}=0$ and then $q_{p}(x)=0$ for all $p$ prime to $m$.) On the other hand, since $0=\mathscr{C}_{p, \infty}=\bigcap_{n \geq 0} \mathscr{C}_{p, n}$, when $q_{p}(x) \neq 0$ we have an integer $n=n(x, p) \in \mathbb{N}, n \geq 1$, such that $q_{p}(x) \in \mathscr{C}_{p, n-1} \backslash \mathscr{C}_{p, n}$. Hence $x \in \mathscr{P}_{p, n(p)-1} \backslash \mathscr{P}_{p, n(p)}$. This proves (d).

Finally, by definition of the topology of $\operatorname{Spc}(\mathscr{K})$ (Definition 1.3) any closed subset is an intersection of supports, $\operatorname{supp}(x)$, for $x \in \mathscr{K}$. It is easy to check that they are the announced ones. The only subtlety is the appearance of the closed point $\left\{\mathscr{P}_{p, \infty}\right\}$ which is not the support of any object but which is $\bigcap_{n \geq 1} \overline{\left\{\mathscr{P}_{p, n}\right\}}$. 
Remark 9.6 We observe that $\mathrm{Spc}\left(\mathrm{SH}^{\mathrm{fin}}\right)$ is not a noetherian topological space since, for any $p \in \mathbb{P}$, the nonempty family of closed subsets $\left\{\overline{\left\{\mathscr{P}_{p, n}\right\}} \mid 0 \leq n<\infty\right\}$ has no minimal element, its intersection being $\left\{\mathscr{P}_{p, \infty}\right\}$. In particular, $\mathrm{Spc}\left(\mathrm{SH}^{\mathrm{fin}}\right)$ cannot be realized as the homogeneous spectrum $\operatorname{Spec}^{\mathrm{h}}\left(R^{\bullet}\right)$ of a noetherian graded ring $R^{\bullet}$.

Proposition 9.7 The locally ringed space $\mathrm{Spec}\left(\mathrm{SH}^{\mathrm{fin}}\right)$ is not a scheme. Nor is any of the local ones $\operatorname{Spec}\left(\mathrm{SH}_{(p)}^{\mathrm{fin}}\right)$, for any prime $p$.

Proof Let $p \in \mathbb{P}$ be a prime. We know by Corollary 9.2 that $\mathrm{SH}_{(p)}^{\mathrm{fin}}$ is a local category and its spectrum $\mathrm{Spc}\left(\mathrm{SH}_{(p)}^{\mathrm{fin}}\right)$ is a local topological space. Hence, if the ringed space $\operatorname{Spec}\left(\mathrm{SH}_{(p)}^{\mathrm{fin}}\right)$ was a scheme, it would be the spectrum of the local ring of global sections of its structure sheaf, which is just $\mathbb{Z}_{(p)}$. Obviously, the spectrum of $\mathrm{SH}_{(p)}^{\mathrm{fin}}$ is much bigger than $\operatorname{Spec}\left(\mathbb{Z}_{(p)}\right)=\{(0),(p)\}$. The global result follows, as a scheme remains a scheme locally.

Remark 9.8 The category $\mathrm{SH}_{(p)}^{\mathrm{fin}}$ only has trivial invertible objects, so even the twisted version of $\rho_{\mathscr{K}}^{\bullet}$ cannot be injective. In conclusion, the triangular spectrum $\operatorname{Spc}(\mathscr{K})$ considerably differs from the algebraic spectra $\operatorname{Spec}\left(\mathrm{R}_{\mathscr{K}}\right)$ or $\operatorname{Spec}^{\mathrm{h}}\left(\mathrm{R}_{\mathscr{K}}^{\bullet}\right)$ in general. This of course indicates that $\operatorname{Spc}(\mathscr{K})$ is a better, finer invariant than both $\operatorname{Spec}^{\mathrm{h}}\left(\mathrm{R}_{\mathscr{K}}^{\bullet}\right)$ and $\operatorname{Spec}\left(\mathrm{R}_{\mathscr{K}}\right)$.

\section{Spectra of motivic spectra}

For this section, let $\mathscr{K}=\mathscr{K}^{\mathbb{A}^{1}}:=\left(\mathrm{SH}_{F}^{\mathbb{A}^{1}}\right)^{\mathrm{c}}$ be the subcategory of compact objects in the Morel-Voevodsky stable $\mathbb{A}^{1}$-homotopy category $\mathrm{SH}_{F}^{\mathbb{A}^{1}}$ over a field $F$; see Voevodsky [33] and Morel [24]. This category can be defined over a more general basis but the computation of $\operatorname{End}_{\mathscr{K}}(\mathbb{1})$, which we use below, is only performed for a field so far. The category $\mathscr{K}^{\mathbb{A}^{1}}$ is connective in the sense of Definition 7.6, ie $\operatorname{Hom}_{\mathscr{K}}\left(\mathbb{1}, \Sigma^{i}(\mathbb{1})\right)=0$ for $i>0$; see [33, Theorem 4.14], [25, Section 6] or [26, Corollary 4.3.3]. The beautiful computation of $\operatorname{End}_{\mathscr{K}}(\mathbb{1})$ is due to Morel (see [25, Theorem 6.2.1]):

$$
\operatorname{End}_{\mathscr{K ^ { A }}} \mathbb{A}^{1}(\mathbb{1}) \cong \mathrm{GW}(F) .
$$

This holds at least when the field $F$ is perfect and of characteristic different from two, which we assume from now on. The ring $\mathrm{GW}(F)$ is the Grothendieck-Witt ring of quadratic forms over $F$, that is, the group completion of the abelian semiring of isometry classes of nondegenerate quadratic forms over $F$, with orthogonal sum as addition and tensor product as multiplication. This ring is classical and its quotient $\mathrm{W}(F)=\mathrm{GW}(F) / h$ by the 2 -dimensional hyperbolic plane $h=\langle 1,-1\rangle$ is the even more classical Witt ring; see Lam [18]. Applying our Theorem 7.13, we get: 
Corollary 10.1 The map $\rho_{\mathscr{K}}: \operatorname{Spc}\left(\mathscr{K}^{\mathbb{A}^{1}}\right) \rightarrow \operatorname{Spec}(\mathrm{GW}(F))$ is surjective.

Remark 10.2 The latter spectrum $\operatorname{Spec}(\operatorname{GW}(F))$ is well-known, at least for the Witt group part. Let us remind the reader.

There is always a ring homomorphism, rank: $\mathrm{GW}(F) \rightarrow \mathbb{Z}$, induced by the rank of quadratic forms. For instance, rank: $\mathrm{GW}(F) \cong \mathbb{Z}$ is an isomorphism for $F$ quadratically closed, like $F=\mathbb{C}$. On the other hand, if there exists an ordering $\alpha$ on $F$ (equivalently, if -1 is not a sum of squares in $F$ ) there is another ring homomorphism, $\operatorname{sgn}_{\alpha}: \mathrm{GW}(F) \rightarrow \mathbb{Z}$, induced by the signature of quadratic forms (for $a \in F^{\times}$, we have $\operatorname{sgn}_{\alpha}(\langle a\rangle)=1$ if $a>0$ with respect to the ordering $\alpha$ and $\operatorname{sgn}_{\alpha}(\langle a\rangle)=-1$ otherwise). Using this, one can show for instance that $\operatorname{Spec}(\mathrm{GW}(\mathbb{R}))$ is equal to two copies of $\operatorname{Spec}(\mathbb{Z})$ attached together at the point $2 \mathbb{Z}$.

More generally, the above surjective ring homomorphisms, rank: $\mathrm{GW}(F) \rightarrow \mathbb{Z}$ and $\operatorname{sgn}_{\alpha}: \operatorname{GW}(F) \rightarrow \mathbb{Z}$, yield copies of $\operatorname{Spec}(\mathbb{Z})$ as closed subsets of $\operatorname{Spec}(\operatorname{GW}(F))$.

(a) For $q \in \operatorname{Spec}(\mathbb{Z})$ define the ideal $\mathfrak{q}_{q}:=\operatorname{rank}^{-1}(q)$. The ideal $I:=\mathfrak{q}_{2}=\{x \in$ $\mathrm{GW}(F) \mid \operatorname{rank}(x)$ is even\} is called the fundamental ideal.

(b) For every ordering $\alpha$ on $F$ (if any) and for every prime $p \in \operatorname{Spec}(\mathbb{Z})$, define the ideal $\mathfrak{p}_{\alpha, p}:=\left(\operatorname{sgn}_{\alpha}\right)^{-1}(p)$.

One has $I=\mathfrak{p}_{\alpha, 2}$ for every $\alpha$ and there is no other redundancy in the above list:

$$
\operatorname{Spec}(\operatorname{GW}(F))=\left\{\mathfrak{q}_{q} \mid q \in \operatorname{Spec}(\mathbb{Z})\right\} \cup \underset{\alpha \text { ordering }}{\bigcup}\left\{\mathfrak{p}_{\alpha, p} \mid p \in \operatorname{Spec}(\mathbb{Z}) \backslash\{2\}\right\} .
$$

Figure 1 shows a picture of $\operatorname{Spec}(\operatorname{GW}(F))$. In the figure, the lines $\circ-\square$ and $\circ \cdots \square$ indicate inclusions $\circ \subset \square$, that is, $\square$ is in the closure of $\circ$. These are the only inclusions. The dotted part, beyond $I$, appears only when $F$ has at least one ordering otherwise $\operatorname{Spec}(G W(F))=\operatorname{Spec}(\mathbb{Z})$. This copy of $\operatorname{Spec}(\mathbb{Z})$, coming via the rank, is the only one which is always present.

To prove this, first recall the spectrum of $\mathrm{W}(F)=\mathrm{GW}(F) / h$ where $h=\langle 1,-1\rangle$, from Harrison and Lorenz-Leicht [19]. It can be found in [18, Section VIII.7, page 277] and corresponds exactly to the dotted part of 1 , including $I$. It just remains to describe $\operatorname{Spec}(\operatorname{GW}(F)[1 / h])$ and this is immediate from $\operatorname{GW}(F)[1 / h] \simeq \mathbb{Z}[1 / 2]$. The latter is induced by the rank and uses the equation $\langle a\rangle \otimes h=\langle a,-a\rangle=\langle 1,-1\rangle=h$ in $\operatorname{GW}(F)$ (which holds in any characteristic). Note that the induced topology on the subspace $\left\{\mathfrak{p}_{\alpha, 0} \mid \alpha\right.$ ordering on $\left.F\right\}$ is homeomorphic to the so-called Harrison topology on the set of orderings; see [18, Corollary 7.6, page 279]. Being a Boolean space, it is not discrete unless it is finite. 


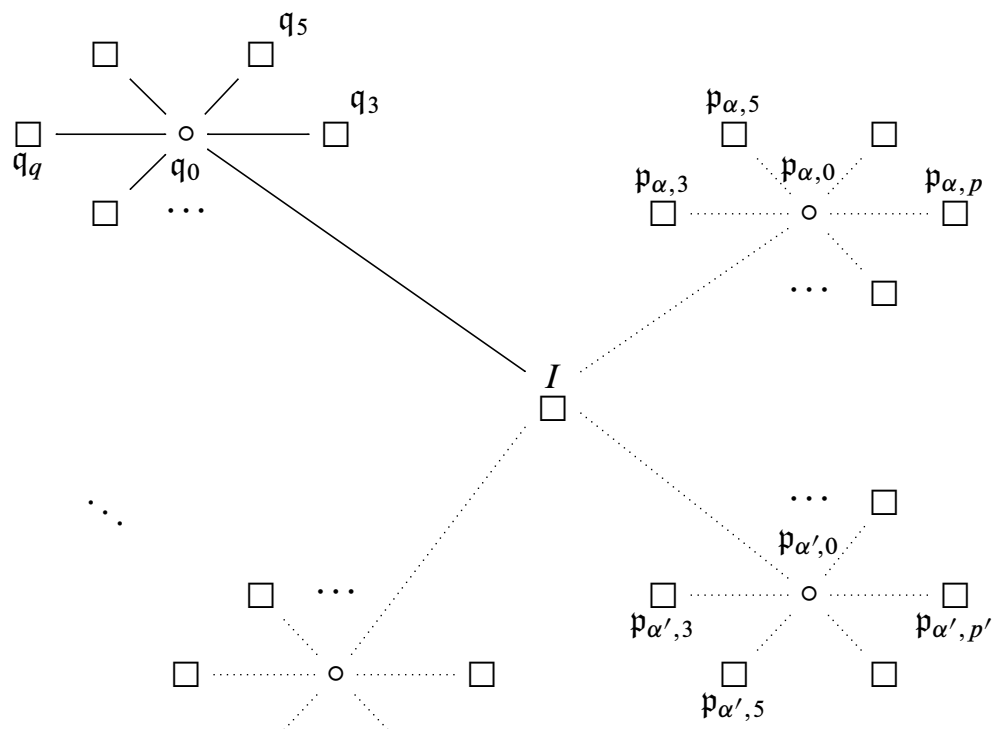

Figure 1

Remark 10.3 We now wonder what lives "above" this space $\operatorname{Spec}(\operatorname{GW}(F))$, that is, how much bigger is the spectrum of our $\mathscr{T}^{\mathbb{A}^{1}}=\left(\mathrm{SH}_{F}^{\mathbb{A}^{1}}\right)^{\mathrm{c}}$, compared to $\operatorname{Spec}(\mathrm{GW}(F))$. The first answer is coming from $\mathrm{SH}^{\text {fin }}$. Let us assume that $F \subset \mathbb{C}$ has a complex embedding. Then we have two $\otimes$-triangulated functors

$$
\mathrm{SH}^{\mathrm{fin}} \longrightarrow \mathscr{K}^{\mathbb{A}^{1}} \stackrel{\mathrm{Re}}{\longrightarrow} \mathrm{SH}^{\mathrm{fin}},
$$

the first one being the constant one and the second being complex realization. Their composition is the identity. So, there is a copy of $\operatorname{Spc}\left(\mathrm{SH}^{\mathrm{fin}}\right)$, as described in Section 9, embedded inside $\operatorname{Spc}\left(\mathscr{K}^{\mathbb{A}^{1}}\right)$ via $\operatorname{Spc}(\operatorname{Re})$. On the level of central rings, this corresponds to the copy of $\operatorname{Spec}(\mathbb{Z})$ in $\operatorname{Spec}(\mathrm{GW}(F))$ given by the rank. This shows that the fibers of $\rho_{\mathscr{K}}$ are at least as big as in topology.

As should be expected, there is even more here than in topology:

Proposition 10.4 The split inclusion $\mathrm{Spc}(\mathrm{Re}): \mathrm{Spc}\left(\mathrm{SH}^{\mathrm{fin}}\right) \rightarrow \operatorname{Spc}\left(\mathscr{K}^{\mathbb{A}^{1}}\right)$ is not surjective. Its image is contained in the proper closed subset $\operatorname{supp}(\operatorname{cone}(\eta))$, where $\eta \in \operatorname{Hom}_{\mathscr{H}}\left(\mathbb{1}, \mathbb{G}_{\mathrm{m}}^{\otimes-1}\right)$ is the algebraic Hopf map. This closed subset is also $\operatorname{supp}\left(\mathbb{P}^{2}\right)$.

Proof The algebraic Hopf map is given by the natural map $\mathbb{A}_{F}^{2} \backslash\{0\} \rightarrow \mathbb{P}_{F}^{1}$ and the facts that $\mathbb{A}_{F}^{2} \backslash\{0\} \cong \Sigma\left(\mathbb{G}_{\mathrm{m}}^{\otimes 2}\right)$ and $\mathbb{P}_{F}^{1} \cong \Sigma\left(\mathbb{G}_{\mathrm{m}}\right)$. See for instance Morel [25, 
Section 6.1]. Its complex realization $\operatorname{Re}(\eta)$ is the topological Hopf map, which is $\otimes$-nilpotent. However, Morel proves that the algebraic $\eta$ is not nilpotent; see [25, Corollary 6.4.5, page 258]. We then use two general results:

(a) For any $\otimes$-triangulated functor $F: \mathscr{K} \rightarrow \mathscr{L}$, if we $\varphi=\operatorname{Spc}(F): \operatorname{Spc}(\mathscr{L}) \rightarrow \operatorname{Spc}(\mathscr{K})$ denotes the induced map and if $x \in \mathscr{K}$, then $\operatorname{supp}(F(x))=\varphi^{-1}(\operatorname{supp}(x))$ in $\operatorname{Spc}(\mathscr{L})$; see [1, Proposition 3.6]. Hence $\varphi(\operatorname{supp}(F(x))) \subset \operatorname{supp}(x)$.

(b) By Theorem 2.15 above, for any $\otimes$-invertible object $u \in \mathscr{K}$, a morphism $f: \mathbb{1} \rightarrow u$ is $\otimes$-nilpotent if and only if $\langle\operatorname{cone}(f)\rangle=\mathscr{K}$. The latter is equivalent to the equality $\operatorname{supp}(\operatorname{cone}(f))=\operatorname{Spc}(\mathscr{K})$; see [1, Corollary 2.5].

In our case, (b) and the nilpotence of $\operatorname{Re}(\eta)$ imply that $\operatorname{supp}(\operatorname{Re}(\operatorname{cone}(\eta)))=\operatorname{Spc}\left(\operatorname{SH}^{\mathrm{fin}}\right)$. Hence by (a), we get $\operatorname{Im}(\operatorname{Spc}(\operatorname{Re})) \subset \operatorname{supp}(\operatorname{cone}(\eta))$, as announced. The latter is smaller than $\operatorname{Spc}\left(\mathscr{K}^{\mathbb{A}^{1}}\right)$ by $(\mathrm{b})$ and the non-nilpotence of the algebraic $\eta$. Finally iscone $(\eta)=\mathbb{P}^{2}$ can be seen from the Mayer-Vietoris square

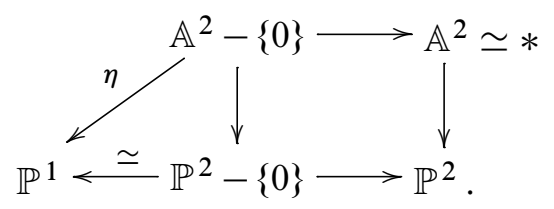

(All schemes are pointed, eg at the points corresponding to $[1: 1: 1] \in \mathbb{P}^{2}$.)

Remark 10.5 There is also a continuous map $\rho_{\mathscr{K}}^{\bullet}: \operatorname{Spc}\left(\mathscr{K}^{\mathbb{A}^{1}}\right) \rightarrow \operatorname{Spec}^{\mathrm{h}}\left(\mathrm{R}_{\mathscr{K}}^{\bullet \mathbb{A}^{1}, u}\right)$ obtained from Section 5 with $u=\mathbb{G}_{\mathrm{m}}$ as $\otimes$-invertible. Morel proves that $\mathrm{R}_{\mathscr{K}^{\mathbb{A}^{1}}, u}^{0} \cong$ $K_{\bullet}^{\mathrm{MW}}(F)$ is the Milnor-Witt $K$-theory of the field $F$; see more in [25, Section 6, page 251]. Further determination of $\operatorname{Spc}\left(\left(\mathrm{SH}_{F}^{\mathrm{A}^{1}}\right)^{\mathrm{c}}\right)$, including the question of the surjectivity of this $\rho_{\mathscr{H}}^{\bullet}$, is a challenging endeavor in which the reader is welcome to engage.

\section{References}

[1] P Balmer, The spectrum of prime ideals in tensor triangulated categories, J. Reine Angew. Math. 588 (2005) 149-168 MR2196732

[2] P Balmer, Supports and filtrations in algebraic geometry and modular representation theory, Amer. J. Math. 129 (2007) 1227-1250 MR2354319

[3] P Balmer, Niveau spectral sequences on singular schemes and failure of generalized Gersten conjecture, Proc. Amer. Math. Soc. 137 (2009) 99-106 MR2439430

[4] P Balmer, Picard groups in triangular geometry and applications to modular representation theory, Trans. Amer. Math. Soc. 362 (2010) 3677-3690 
[5] P Balmer, D J Benson, J F Carlson, Gluing representations via idempotent modules and constructing endotrivial modules, J. Pure Appl. Algebra 213 (2009) 173-193 MR2467395

[6] P Balmer, G Favi, Gluing techniques in triangular geometry, Q. J. Math. 58 (2007) 415-441 MR2371464

[7] D J Benson, Representations and cohomology. I. Basic representation theory of finite groups and associative algebras, second edition, Cambridge Studies in Advanced Math. 30, Cambridge Univ. Press (1998) MR1644252

[8] D Benson, S B Iyengar, H Krause, Local cohomology and support for triangulated categories, Ann. Sci. Éc. Norm. Supér. (4) 41 (2008) 573-619 MR2489634

[9] A B Buan, H Krause, Ø Solberg, Support varieties: an ideal approach, Homology, Homotopy Appl. 9 (2007) 45-74 MR2280286

[10] J M Cohen, Coherent graded rings and the non-existence of spaces of finite stable homotopy type, Comment. Math. Helv. 44 (1969) 217-228 MR0247628

[11] I Dell'Ambrogio, Prime tensor ideals in some triangulated categories of $C^{*}$-algebras, $\mathrm{PhD}$ thesis, ETH Zürich (2008)

[12] M E Harris, Some results on coherent rings, Proc. Amer. Math. Soc. 17 (1966) 474-479 MR0193111

[13] R Hartshorne, Algebraic geometry, Graduate Texts in Math. 52, Springer, New York (1977) MR0463157

[14] M Hochster, Prime ideal structure in commutative rings, Trans. Amer. Math. Soc. 142 (1969) 43-60 MR0251026

[15] M J Hopkins, J H Smith, Nilpotence and stable homotopy theory. II, Ann. of Math. (2) 148 (1998) 1-49 MR1652975

[16] M Hovey, J H Palmieri, N P Strickland, Axiomatic stable homotopy theory, Mem. Amer. Math. Soc. 128 (1997) x+114 MR1388895

[17] A Krishna, Perfect complexes on Deligne-Mumford stacks and applications, J. KTheory 4 (2009) 559-603 MR2570954

[18] T Y Lam, Introduction to quadratic forms over fields, Graduate Studies in Math. 67, Amer. Math. Soc. (2005) MR2104929

[19] F Lorenz, J Leicht, Die Primideale des Wittschen Ringes, Invent. Math. 10 (1970) 82-88 MR0266949

[20] A Lorenzini, Graded rings, from: “The curves seminar at Queens, Vol. II (Kingston, Ont., 1981/1982)", (A V Geramita, editor), Queen’s Papers in Pure and Appl. Math. 61, Queen's Univ., Kingston, ON (1982) Exp. No. D, 41 MR783088

[21] S Mac Lane, Categories for the working mathematician, second edition, Graduate Texts in Math. 5, Springer, New York (1998) MR1712872 
[22] H R Margolis, Spectra and the Steenrod algebra. Modules over the Steenrod algebra and the stable homotopy category, North-Holland Math. Library 29, North-Holland, Amsterdam (1983) MR738973

[23] R Meyer, Categorical aspects of bivariant $K$-theory, from: " $K$-theory and noncommutative geometry”, (G Cortiñas, J Cuntz, M Karoubi, R Nest, C A Weibel, editors), EMS Ser. Congr. Rep., Eur. Math. Soc., Zürich (2008) 1-39 MR2513331

[24] F Morel, An introduction to $\mathbb{A}^{1}$-homotopy theory, from: "Contemporary developments in algebraic $K$-theory", (M Karoubi, A O Kuku, C Pedrini, editors), ICTP Lect. Notes XV, Abdus Salam Int. Cent. Theoret. Phys., Trieste (2004) 357-441 MR2175638

[25] F Morel, On the motivic $\pi_{0}$ of the sphere spectrum, from: "Axiomatic, enriched and motivic homotopy theory", (J P C Greenlees, editor), NATO Sci. Ser. II Math. Phys. Chem. 131, Kluwer Acad. Publ., Dordrecht (2004) 219-260 MR2061856

[26] F Morel, The stable $\mathbb{A}^{1}$-connectivity theorems, $K$-Theory 35 (2005) 1-68 MR2240215

[27] A Neeman, Triangulated categories, Annals of Math. Studies 148, Princeton Univ. Press (2001) MR1812507

[28] N C Phillips, Equivariant $K$-theory and freeness of group actions on $C^{*}$-algebras, Lecture Notes in Math. 1274, Springer, Berlin (1987) MR911880

[29] D C Ravenel, Nilpotence and periodicity in stable homotopy theory, Annals of Math. Studies 128, Princeton Univ. Press (1992) MR1192553 Appendix C by J Smith

[30] J Rickard, Derived categories and stable equivalence, J. Pure Appl. Algebra 61 (1989) 303-317 MR1027750

[31] G Segal, The representation ring of a compact Lie group, Inst. Hautes Études Sci. Publ. Math. (1968) 113-128 MR0248277

[32] J-L Verdier, Des catégories dérivées des catégories abéliennes, Astérisque 239, Soc. Math. France, Paris (1996) MR1453167 With a preface by L Illusie, Edited and with a note by $\mathrm{G}$ Maltsiniotis

[33] V Voevodsky, $\mathbb{A}^{1}$-homotopy theory, from: "Proceedings of the International Congress of Mathematicians, Vol. I (Berlin, 1998)", Doc. Math. Extra Vol. I (1998) 579-604 MR1648048

Mathematics Department, UCLA

Box 951555, Los Angeles 90095-1555, United States

balmer@math . ucla.edu

http://www. math.ucla.edu/ balmer

Received: 28 May 2009 Revised: 26 March 2010 